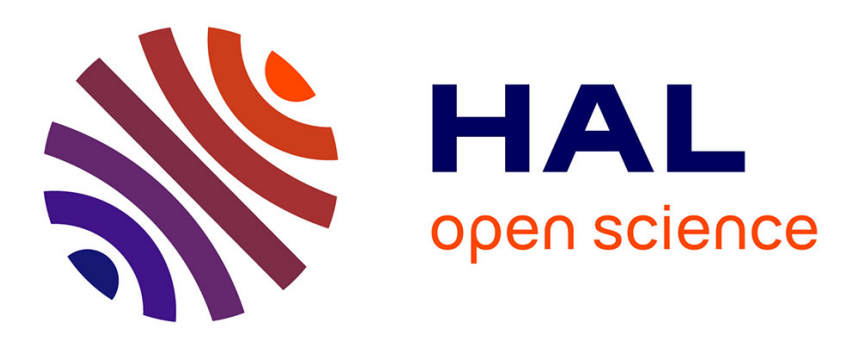

\title{
Dendritic needle network modeling of the Columnar-to-Equiaxed Transition. Part II: three dimensional formulation, implementation and comparison with experiments
}

Chih-Hung Chen, Amirhossein Molavi Tabrizi, Pierre-Antoine Geslin, Alain Karma

\section{To cite this version:}

Chih-Hung Chen, Amirhossein Molavi Tabrizi, Pierre-Antoine Geslin, Alain Karma. Dendritic needle network modeling of the Columnar-to-Equiaxed Transition. Part II: three dimensional formulation, implementation and comparison with experiments. Acta Materialia, 2021, 202, pp.463-477. 10.1016/j.actamat.2020.10.012 . hal-03066632

\section{HAL Id: hal-03066632 https://hal.science/hal-03066632}

Submitted on 15 Dec 2020

HAL is a multi-disciplinary open access archive for the deposit and dissemination of scientific research documents, whether they are published or not. The documents may come from teaching and research institutions in France or abroad, or from public or private research centers.
L'archive ouverte pluridisciplinaire HAL, est destinée au dépôt et à la diffusion de documents scientifiques de niveau recherche, publiés ou non, émanant des établissements d'enseignement et de recherche français ou étrangers, des laboratoires publics ou privés. 


\title{
Dendritic Needle Network Modeling of the Columnar-to-Equiaxed Transition Part II: Three Dimensional Formulation, Implementation and Comparison With Experiments
}

\author{
Chih-Hung Chen ${ }^{\mathrm{a}, *}$, Amirhossein Molavi Tabrizi ${ }^{\mathrm{b}}$, Pierre-Antoine Geslin ${ }^{\mathrm{c}}$, Alain Karma ${ }^{\mathrm{b}, *}$ \\ ${ }^{a}$ Institute of Applied Mechanics, National Taiwan University, Taipei 106, Taiwan \\ ${ }^{b}$ Department of Physics and Center for Interdisciplinary Research on Complex Systems, Northeastern University, Boston, MA 02115, USA \\ ${ }^{c}$ Univ. Lyon, INSA-Lyon, MATEIS UMR 5510 CNRS, 69632 Villeurbanne, France
}

\begin{abstract}
The dendritic needle network (DNN) model tracks the diffusion-controlled growth of branches in the hierarchically structured dendritic network, thereby bridging the well-separated scales traditionally simulated by phase-field and coarse-grained models. In particular, the DNN model relaxes the assumptions on the dendrite growth kinetics and grain structures commonly used in the coarse-grained models. In part I of this paper, we proposed a two-dimensional (2D) version of the DNN model and applied it to investigate the Columnar-to-Equiaxed Transition (CET) to clarify the influence of these assumptions on the prediction of the CET. In order to overcome the limitations inherent in a 2D model, part II presents here a fully three-dimensional (3D) version of the DNN model and its application to the CET. After validation of the 3D model, we perform simulations to study the solidification of Al-7wt.\%Si alloy and compare the results to the experimental measurements conducted on board of the International Space Station (ISS) in the framework of the CET in SOLidification processing project. The comparison shows that the present 3D DNN model is able to provide quantitative prediction of the position and the type of CET at experimental time and lengthscales, without any adjustable parameters.
\end{abstract}

\section{Introduction}

Solidification often leads to complex dendritic microstructures; in particular, a transition from columnar to equiaxed grains is commonly observed in industrial casting processes. The solidification starts with the development of columnar dendrites from the surface of the mold driven by high temperature gradients. The growth of the columnar front is later blocked by the presence of equiaxed grains, which nucleate and grow ahead of the columnar front under lower temperature gradient and slow solidification rate. Since the Columnar-to-Equiaxed transition (CET) greatly influences the grain microstructure and therefore the mechanical performance of the casting products, it became an important challenge in metallurgical sciences to predict precisely the position of the CET and the morphology of the grains.

Over the past decades, several physically-based numer-

\footnotetext{
${ }^{*}$ Corresponding authors

Email addresses: chchen@iam.ntu.edu.tw (Chih-Hung Chen), a.karma@northeastern.edu (Alain Karma)
}

ical models have been developed to study the CET. At the microscopic scale, the phase-field approach for solidification was proposed to model quantitatively the freeboundary problem associated with the movement of the solid-liquid interface $[1,2,3]$. Its main advantage relies on the ability to account naturally for complex morphological and topological changes of the solid-liquid interface encountered in solidification processes. However, this microscopic approach is limited to small system sizes, and is thus far beyond the capacity to address the CET using the phase-field approach. On the other hand, meso-scale models such as volume-averaged based models $[4,5,6]$ and the Cellular Automaton Finite Element (CAFE) method [7, 8] allow to investigate the CET at relevant length-scales but these methods rely on several assumptions regarding the dendrite growth kinetics and grain structures. As shown in part I [9], some of these assumptions becomes invalid for the study of CET, therefore casting doubt on the accuracy of these meso-scale models.

The Dendritic Needle Network (DNN) model has been developed over the past decade $[10,11,12,13]$ to bridge the scales between phase-field and meso-scale ap- 
proaches, while relying on a minimal set of assumptions. In the DNN model, the dendritic microstructure is described as a set of needles represented as parabolas in 2D and paraboloids in 3D. The dynamics of each dendritic needle is prescribed by two conditions that determine uniquely the evolution of its tip velocity and tip radius. Solving these two conditions allows the DNN method to describe both the transient and the steady-state growth of dendrites while accounting for solutal interactions between grains. Since the DNN model overcomes the length and time-scale limitations of the phase-field approach without relying on the assumptions used in the meso-scale models, it becomes an appropriate approach to investigate quantitatively the CET that occurs on the centimeter length-scale.

In part I of this paper [9], we present a 2D formulation of the DNN model and its application to investigate the CET. We demonstrate that the 2D formulation is able to describe all the key aspects of the CET and allows to examine the assumptions commonly used in the analytical and coarse-grained models. We observed in simulations that the columnar front has a higher undercooling than predicted by the steady-state Ivantsov solution due to interactions with neighboring dendrites and higherorder branches. Our simulation results also suggest that the steady-state growth assumption commonly used in the coarse-grained approaches is inaccurate and fails to describe quantitatively the growth of equiaxed grains. However, since the scaling laws for the transient and steadystate dendritic growth are different in $2 \mathrm{D}$ and $3 \mathrm{D}$, a fully 3D DNN model is required to study the CET quantitatively.

In this part II of the paper, we present the extension of the DNN formulation from 2D to 3D. Building on the previous formulations $[11,12,13]$, the model also incorporates two features necessary to model the CET: the nucleation of new grains and the growth of needles of arbitrary orientations. We then apply the 3D DNN approach to investigate the CET on experimental length and time-scales in order to compare quantitatively our simulations results to the experimental observations obtained in the framework of the Columnar-to-Equiaxed Transition in SOLidification processing (CETSOL) project [14, 15, 16, 17]. All simulations in this study are performed using the parameters pertaining to $\mathrm{Al}-7 \mathrm{wt} . \% \mathrm{Si}$ alloy used in the CETSOL project.

This part II is organized as follows: Section 2 discusses the formulation of the 3D DNN model to study CET. Section 3 presents quantitative validations of the DNN model against the steady-state Ivantsov solution. Section 4 discusses the stability of the columnar front and the side- branching dynamics. The nucleation of grains in the DNN model and the follow-up grain growth are discussed in Section 5. Section 6 is dedicated to the DNN modeling of the CET and the comparison with CETSOL experiments. Finally, we summarize our findings in Section 7 and provide possible extensions of this study.

\section{3D Dendritic Needle Network model}

\subsection{Sharp-interface solidification model}

We consider the solidification of a dilute binary alloy in a purely diffusive regime without convection, and the solute concentration in the melt is described by the Fick diffusion equation

$$
\frac{\partial c}{\partial t}=D \nabla^{2} c,
$$

where $D$ is the solute diffusion coefficient in the liquid. By neglecting the diffusivity in the solid, the solute mass conservation at the solid-liquid interface is described by the Stefan condition $[18,19]$

$$
(1-k) c_{l} v_{n}=-\left.D \partial_{n} c\right|_{i},
$$

where $c_{s}$ and $c_{l}$ are the equilibrium concentration in the solid and in the liquid obtained from the alloy phase diagram, respectively, $k=c_{s} / c_{l}$ is the solute partition coefficient, $v_{n}$ is the solid-liquid interface velocity (normal to the interface), and $\left.\partial_{n} c\right|_{i}$ represents the normal solute concentration gradient at the solid/liquid interface.

Because the thermal diffusivity is two to four orders of magnitude greater than the solute diffusivity in the liquid [20], the temperature field is assumed to reach equilibrium instantaneously on the characteristic time-scale of solute diffusion. Thus, the temperature field in this model is directly imposed by the external thermal condition without considering the thermal diffusion. The solid-liquid interface is in local thermodynamic equilibrium, and the concentration on the liquid side of the interface $c_{l}$ is related to the interface temperature through the Gibbs-Thomson relation [21]:

$$
T=T_{M}+m_{l} c_{l}-\Gamma f(\theta) \kappa,
$$

where $T_{M}$ is the melting point of pure solvent, $m_{l}$ is the liquidus slope $\left(m_{l}<0\right), \Gamma=\gamma_{0} T_{M} / L_{h}$ is the GibbsThomson coefficient, $L_{h}$ is the latent heat of fusion per unit volume, $\kappa$ is the interface curvature and $f(\theta)$ describes the dependence of the surface tension on the orientation $\theta$. The composition of the alloy far from the solid is imposed by a far-field boundary condition, $c \rightarrow c_{\infty}$ where $c_{\infty}$ is the nominal composition of the alloy. 
For interface radii much larger than the tip radius $\rho$, the surface tension effects become negligible, and Eq. (3) becomes

$$
T(\mathbf{x})=T_{M}+m_{l} c_{l}(\mathbf{x}),
$$

and thus the liquidus temperature of the alloy is

$$
T_{L}=T_{M}+m_{l} c_{\infty}
$$

In order to obtain a better understanding of the CET, we use in this study the experimental temperature data as the input data for the thermal conditions instead of using Bridgman set-up simplifications.

Based on Eq. (4) and Eq. (5), we obtain

$$
c_{l}(\mathbf{x})=c_{\infty}+\frac{T(\mathbf{x})-T_{L}}{m_{l}} .
$$

To simplify the numerical implementation and the following discussion, we choose the solidus temperature $T_{0}$ as the reference temperature, and let $c_{l}^{0}=c_{\infty} / k$ represent the liquid equilibrium composition of a flat interface at $T_{0}$. Thus, we have

$$
T_{0}=T_{M}+m_{l} c_{l}^{0}
$$

For convenience, we define a reduced solute field using the following relation:

$$
u(\mathbf{x}) \equiv \frac{c_{l}^{0}-c(\mathbf{x})}{(1-k) c_{l}^{0}} .
$$

From Eqs. (5) to (8), the reduced concentration on the liquid side of the solid-liquid interface is:

$$
u_{l}(\mathbf{x})=\frac{T_{0}-T(\mathbf{x})}{T_{0}-T_{L}} .
$$

Note that the condition $c \rightarrow c_{\infty}$ far from the solid becomes $u \rightarrow 1$.

We rewrite the diffusion equation in Eq. (1) and the Stefan condition in Eq. (2) in terms of the reduced solute field:

$$
\begin{aligned}
\frac{\partial u}{\partial t} & =D \nabla^{2} u, \\
q v_{n} & =\left.D \partial_{n} u\right|_{i},
\end{aligned}
$$

where $q \equiv c_{l} / c_{l}^{0}$.

Let $T^{\text {tip }}$ represent the temperature at a dendritic tip. Then from Eq. (6) the solute concentration at the tip $c_{l}^{\text {tip }}$ is:

$$
c_{l}^{\mathrm{tip}}=c_{\infty}+\frac{T^{\mathrm{tip}}-T_{L}}{m_{l}} .
$$

The supersaturation at the tip (tip supersaturation in the non-dimensional form) is defined as $[22,10]$ :

$$
\Omega^{\mathrm{tip}}=\frac{c^{\mathrm{tip}}-c_{\infty}}{(1-k) c^{\mathrm{tip}}} .
$$

A local capillary length $d^{\text {tip }}$ on the tip scale is defined as $[10,13]$

$$
d^{\text {tip }}=\frac{\Gamma}{\left|m_{l}\right|(1-k) c_{l}^{\text {tip }}} .
$$

The capillarity length $d_{0}$ at the reference (solidus) temperature $T_{0}$ is chosen as a reference capillarity length:

$$
d_{0}=\frac{\Gamma}{\left|m_{l}\right|(1-k) c_{l}^{0}} .
$$

Finally, we note that $q^{\text {tip }}=c_{l}^{\text {tip }} / c_{l}^{0}$, so the reference and tip capillarity lengths are related through:

$$
d_{0}=d^{\text {tip }} q^{\text {tip }}
$$

\subsection{Dendritic needle network model}

The dendritic needle network (DNN) model has been discussed in details in 2D [10,9] and has been recently extended to 3D [11, 12], so we here only present an overview of the approach.

The DNN model describes the dendritic grain structure as an array of parabloid needles, and each needle grows along a specific constant direction $\hat{t}$. In the following discussion, the growth direction of the needles is represented in a polar coordinate system as displayed in Fig. 1: $\theta, \phi$ represent the polar and azimuthal angles of the growth direction, respectively so that $\hat{t}=$ $(\sin \theta \cos \phi, \sin \theta \sin \phi, \cos \theta)$.

The dynamic of the dendritic tips in the DNN model, which is prescribed by the tip radius and the tip velocity (see Fig. 1), strongly depend on the surrounding solutal environment and this approach captures the interactions between branches in both transient and steady-state growth regimes. The growth velocity $V_{t i p}$ and the tip radius $\rho$ of each needle are uniquely determined by combining two independent conditions: (i) a solvability condition controlled by the strength of the surface tension anisotropy, and (ii) a mass conservation condition given by a flux intensity factor. The first condition is applied at the scale of the tip radius, and the second one is calculated at an intermediate scale between the tip radius and the diffusion length.

\subsubsection{Solvability condition at the tip}

At the scale of the tip radius, the solvability condition relates the product $\rho^{2} V_{\text {tip }}$ to the strength of the surface tension anisotropy $[23,24,25]$ through the following relation

$$
\rho^{2} V_{t i p}=\frac{2 D d^{\mathrm{tip}}}{\sigma}
$$

where the tip selection parameter $\sigma$ is obtained by the solid/liquid surface tension anisotropy. The validity of 
Eq. (16) has been checked in phase-field simulations [27] by showing that after a short transient, the product $\rho^{2} V_{t i p}$ reaches a constant value during dendritic growth.

Let $\rho_{s}$ and $V_{s}$ represent the steady-state tip radius and velocity, respectively. The tip Péclet number is defined as $\mathrm{Pe} \equiv \rho_{s} V_{s} /(2 D)[18,28]$. The well-known Ivantsov solution relates the supersaturation $\Omega$ and the Péclet number Pe through the relation:

$$
\Omega=\operatorname{Pe} e^{\operatorname{Pe}} \int_{\operatorname{Pe}}^{\infty} \frac{e^{-\tau}}{\tau} d \tau,
$$

Thus, the analytical solutions for $\rho_{s}(\Omega)$ and $V_{s}(\Omega)$ are calculated by solving the microscopic solvability condition Eq. (16) with $\rho=\rho_{s}$ and $V_{\text {tip }}=V_{s}$ together with the Ivantsov relation Eq. (17).

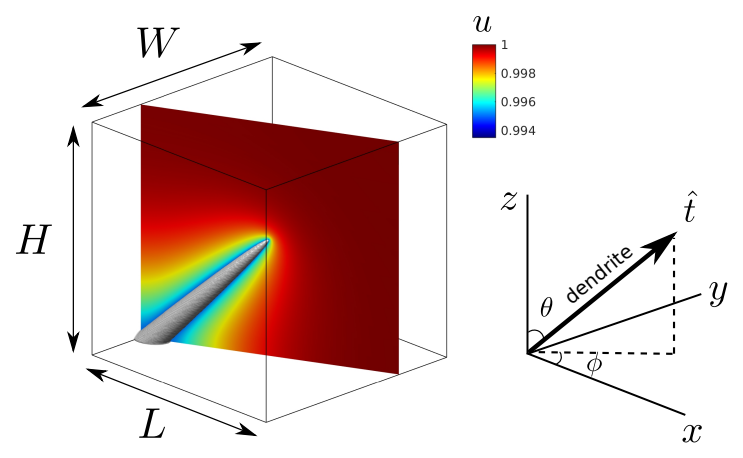

Figure 1: Snapshot of a 3D dendrite growing at $\theta$ and $\phi$ in a 3D DNN simulation of a domain of $L \times W \times H$ with supersaturation $\Omega$. The colormap represents the reduced solute field $u$ (see Eq. (8)). Inset: the dendrite growth direction $\hat{t}$ is written in terms of the polar angle $\theta$ and the azimuthal angle $\phi$, respectively.

\subsubsection{Flux intensity factor $\mathcal{F}$}

At the length scale of the diffusion field, the strength of the solute flux directed towards the dendritic tip is measured by a flux intensity factor denoted by $\mathcal{F}$, which is computed by a surface integration around the tip of each dendrite $[11,12,13]$. To compute $\mathcal{F}$ in our model, we consider a dendrite, described as a paraboloid of tip radius $\rho$, growing along $\hat{t}$ with its tip located inside a cuboid integration box $\Sigma$, as shown in Fig. 2. With no loss of generality and to reduce the complexity of the flux integral calculations, the model only consider that each dendrite pass through one face of their integration box. The plane of intersection is denoted by $S$, and $\hat{n}_{S}$ is defined as the inward unit normal vector of $S$. The six faces of the integration box $\Sigma$ are denoted by $S_{x}^{+}, S_{x}^{-}, S_{y}^{+}, S_{y}^{-}, S_{z}^{+}$, and $S_{z}^{-}$, as shown in Fig. 2a. The choice of which face intersects the dendrite depends on the dendritic direction in terms of $\theta$ and $\phi$. These different conditions are summarized in six categories (C1 - C6) in Table 1.

The height of the box is $2 H_{b o x}$, and during the dendrite growth, the tip is kept at the center of $\Sigma$ along the $\hat{n}_{s}$ direction. Hence, the intersected plane $S$ satisfies the following equation:

$$
S:\left(\vec{r}-\vec{r}_{t i p}\right) \cdot \hat{n}_{s}=-H_{b o x}
$$

For instance, let's consider a C1-needle and thus $\hat{n}_{s}=$ $\hat{z}$, and $S: z-z^{t i p}=-H_{b o x}$ (see Table 1 and Fig. 2a). While the height of the integration box $2 H_{\text {box }}$ is set to be constant during dendrite growth, the length and the width of $\Sigma$ are numerically adjusted in the code (at each timestep) to ensure that $S$ is the only side of the integration box intersecting with the paraboloid during growth. More details on the choice of the integration box dimensions

\begin{tabular}{|c|c|c|c|c|}
\hline Category & $S$ & $\theta$ & $\phi$ & $\hat{n}_{s}$ \\
\hline $\mathrm{C} 1$ & $S_{z}^{+}$ & $0^{\circ} \leq \theta \leq 45^{\circ}$ & $0^{\circ}<\phi \leq 360^{\circ}$ & $\hat{z}$ \\
\hline $\mathrm{C} 2$ & $S_{x}^{+}$ & $45^{\circ}<\theta \leq 135^{\circ}$ & $-45^{\circ}<\phi \leq 45^{\circ}$ & $\hat{x}$ \\
\hline C3 & $S_{y}^{+}$ & $45^{\circ}<\theta \leq 135^{\circ}$ & $45^{\circ}<\phi \leq 135^{\circ}$ & $\hat{y}$ \\
\hline $\mathrm{C} 4$ & $S_{x}^{-}$ & $45^{\circ}<\theta \leq 135^{\circ}$ & $135^{\circ}<\phi \leq 225^{\circ}$ & $-\hat{x}$ \\
\hline C5 & $S_{y}^{-}$ & $45^{\circ}<\theta \leq 135^{\circ}$ & $225^{\circ}<\phi \leq 315^{\circ}$ & $-\hat{y}$ \\
\hline C6 & $S_{z}^{-}$ & $135^{\circ}<\theta \leq 180^{\circ}$ & $0^{\circ}<\phi \leq 360^{\circ}$ & $-\hat{z}$ \\
\hline
\end{tabular}
can be found in Appendix A.

Table 1: The choice of the intersected face of the integration box depends on the needle orientation angles $(\theta, \phi)$, which is summarized by six categories, C1 - C6. $S_{i}^{ \pm}$represents the intersected region of the unit normal vector $\pm \hat{i}$, where $i=\{x, y, z\}$.

In the following discussion, the subscripts $s, l$ and $s l$ represent solid, liquid, and the solid-liquid interface, respectively. The integration box $\Sigma$ is the union of the solid region $\Sigma_{s}$ and the melt region $\Sigma_{l}$, i.e., $\Sigma=\Sigma_{s}+\Sigma_{l}$. The boundary of the domains are denoted $\Gamma=\partial \Sigma$. The integration box intersects the dendrite and the liquid at $\Gamma_{s} \equiv \Sigma_{s} \cap \Sigma$ and $\Gamma_{l} \equiv \Gamma-\Gamma_{s}$, respectively. Thus, we have $\partial \Sigma_{s}=\Gamma_{s}+\Gamma_{s l}$ and $\partial \Sigma_{l}=\Gamma_{l}+\Gamma_{s l}$. Let $\hat{n}_{l}$ and $\hat{n}_{s l}$ represent the unit vectors normal to $\Gamma_{s l}$ and $\Gamma_{l}$, respectively (see Fig. 2b). Thus, the area of the intersected region $\Gamma_{s}$ is given by (see Appendix B):

$$
\mathcal{A}_{s} \equiv \int_{\Gamma_{s}} d \mathcal{A}=\frac{\pi}{\cos \alpha}\left[(\rho \tan \alpha)^{2}+\frac{2 \rho H_{\mathrm{box}}}{\cos \alpha}\right],
$$

where $\alpha \equiv \arccos \left(\hat{t} \cdot \hat{n}_{s}\right)$.

We assume that the shape of the needle is preserved during growth in a moving frame advancing at a velocity $V_{\text {tip }}$ along the growth direction $\hat{t}$. Applying the Stefan condition in Eq. (11) on the dendrite surfaces $\Gamma_{s l}$ yields

$$
q V_{t i p} \hat{t} \cdot \hat{n}_{s l}=D \nabla u \cdot \hat{n}_{s l}
$$


In general, $q$ is a local solutal field dependent quantity, but here the integrate box is chosen small enough (i.e., $\left.H_{b o x}, L_{b o x}, W_{b o x} \ll D / V_{t i p}\right)$ such that we approximate $q \approx$ $q^{\text {tip }}$ in the integration box.

By integrating both sides of Eq. (20) over the surface of dendrite $\Gamma_{s l}$ using the approximation of $q \approx q^{\text {tip }}$, we obtain

$$
q^{\mathrm{tip}} V_{t i p} \int_{\Gamma_{s l}}\left(\hat{t} \cdot \hat{n}_{s l}\right) d \mathcal{A}=D \int_{\Gamma_{s l}}\left(\nabla u \cdot \hat{n}_{s l}\right) d \mathcal{A},
$$

Let's define the flux intensity factor

$$
\mathcal{F} \equiv \frac{1}{2 \pi H_{\mathrm{box}}} \int_{\Gamma_{s l}}\left(\nabla u \cdot \hat{n}_{s l}\right) d \mathcal{A}
$$

and thus Eq. (21) is rewritten as:

$$
q^{\mathrm{tip}} V_{t i p} \int_{\Gamma_{s l}}\left(\hat{t} \cdot \hat{n}_{s l}\right) d \mathcal{A}=2 \pi H_{\mathrm{box}} D \mathcal{F}
$$

We apply the divergence theorem to show that the integration of the divergence of the tip growth direction, $\nabla \cdot \hat{t}$, over the space $\Sigma_{s}$ (see Fig. 2) is

$$
\begin{aligned}
\int_{\Sigma_{s}}(\nabla \cdot \hat{t}) d \mathcal{V} & =\oint_{\partial \Sigma_{s}}(\hat{t} \cdot \hat{n}) d \mathcal{A} \\
& =\int_{\Gamma_{s l}}\left(\hat{t} \cdot \hat{n}_{s l}\right) d \mathcal{A}-\int_{\Gamma_{s}}\left(\hat{t} \cdot \hat{n}_{s}\right) d \mathcal{A} \\
& =\int_{\Gamma_{s l}}\left(\hat{t} \cdot \hat{n}_{s l}\right) d \mathcal{A}-\mathcal{A}_{s} \cos \alpha .
\end{aligned}
$$

By using Eqs. (19), (23), (24), and knowing that $\nabla \cdot \hat{t}=0$, we obtain the dendritic tip velocity:

$$
V_{\text {tip }}=\frac{2 H_{\mathrm{box}} D \mathcal{F}}{\left[(\rho \tan \alpha)^{2}+\frac{2 \rho H_{\mathrm{box}}}{\cos \alpha}\right] q^{\mathrm{tip}}} .
$$

Thus, the growth conditions $\left(\rho, V_{t i p}\right)$ of a needle tip are uniquely determined by combining Eq. (25) with the solvability condition in Eq. (16).

By choosing the needle growing along the computational grid, i.e., $\hat{t}=\hat{n}_{s}$, one can show that Eq. (25) recovers the same flux condition at the tip given in the previously presented 3D DNN model [11]:

$$
\rho V_{\text {tip }}=\frac{1}{q^{\text {tip }}} D \mathcal{F}=\frac{1}{\left[1-(1-k) u^{t i p}\right]} D \mathcal{F} .
$$

(a)

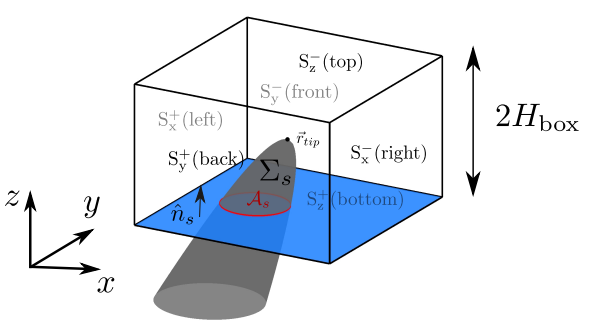

(b)

$$
\partial \Sigma_{l}=\Gamma_{l}+\Gamma_{s l}
$$

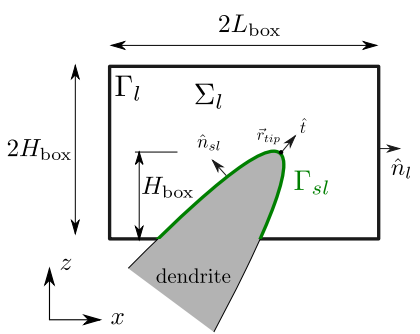

(c)

$$
\partial \Sigma_{s}=\Gamma_{s}+\Gamma_{s l}
$$

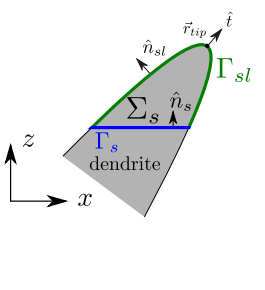

Figure 2: Scheme of a dendritic tip at an intermediate scale between the tip radius $\rho$ and the diffusion length $D / V_{\text {tip }}$. (a) A cuboid integration box is used for computing the FIF of a dendrite growing along $\hat{t}=\left(t_{x}, t_{y}, t_{z}\right)$. The dendrite is described by a paraboloid (in gray) and the size of the integration box is chosen such that the dendrite intersects only one side of the integration box. Let $S$ and $\hat{n}_{S}$ represent the intersected side and its unit normal vector. The region $\Sigma_{s}$ is inside the dendrite and bounded by the closed surfaces $\Gamma_{s}+\Gamma_{s l}$, where $\Gamma_{s}$ is the intersected area of the needle and $S . \Gamma_{s l}$ is the solid-liquid interface between the needle and the melt, and $\hat{n}_{s l}$ is the unit normal vector of $\Gamma_{s l}$. (b) A side view ( $x z$ plane) of the region $\Sigma_{l}$ bounded by closed surfaces $\Gamma_{l}+\Gamma_{s l}$, where $\Gamma_{l}$ refers to the melt region in the integration box. The tip is always at the center of the integration box of constant height $2 H_{\text {box }}$ along the $\hat{n}_{s}$ direction. (c) A side view ( $x z$ plane) of the $\Sigma_{s}$ region.

Hence, the dynamics of the DNN is described by the diffusion equation in Eq. (10) with the imposed boundary conditions on the needles related to the external thermal conditions through Eq. (9) and the condition $u=1$ far from the solid. The time evolution of an individual needle is prescribed by its tip radius $\rho$ and its growth velocity $V_{t i p}$, which are obtained by combining the solvability condition Eq. (16) with the flux condition of Eq. (25) due to mass conservation, where the flux intensity factor $\mathcal{F}$ is given by Eq. (22).

\subsection{Numerical implementation}

The equations of the DNN model are discretized spatially in a finite-difference cubic mesh grid of uniform mesh size $d x$, and solved using an explicit Euler time integration scheme with a constant time step $d t$. The code is 
implemented on Graphics Processing Units (GPUs) with the assistance of the Compute Unified Device Architecture (CUDA) to be computationally efficient.

The main objective of this study is to compare the DNN solutions to experimental observations obtained in the framework of the CETSOL project. However, with the current computer power available, the size of the CETSOL samples are still computationally out of reach of the DNN model. To overcome this limitation, only a relevant region of the experimental domain is simulated numerically. First, simulations are conducted in a slab geometry with height of $H$ and other dimensions of the slab much larger than $H$ to reduce the finite size effect (see Section 3). Second, we implemented a treadmill method to maintain the tip of the most advanced dendrite at the center of the simulation box along the growing direction by adding a new layer of concentration $c=c_{\infty}$ (i.e., $u=1$ ) on the boundary of the box ahead of the dendrites and simultaneously removing the layer on the boundary behind of the dendrites, so the simulation domain size remains constant. We impose no-flux boundary condition for the solute field on all boundaries of the simulation domain.

\section{Quantitative validations}

To validate the model, we first carried out simulations under isothermal conditions at constant temperature $T$ and thus the constant undercooling is

$$
\Delta T=T_{L}-T=\frac{\left|m_{l}\right| c_{\infty}(1-k) \Omega}{(1-(1-k) \Omega)},
$$

where $\Omega=\left(c_{l}-c_{\infty}\right) /\left((1-k) c_{l}\right)$ is the dimensionless supersaturation with $c_{l}$ defined as the liquidus concentration at temperature $T$.

\subsection{Thickness effect on steady-state regime}

Going from 2D to 3D, it is important to examine the influence of the sample thickness on the dynamics of dendritic growth in order to avoid the undesired finite size effect in 3D DNN simulations. Here we apply the DNN method to study the growth of a single dendrite aligned along the computational grid. We employ a slab geometry as shown in Fig. 3a and use the parameters pertaining to $\mathrm{Al}-7 \mathrm{wt} \% \mathrm{Si}$ listed in Table 2.
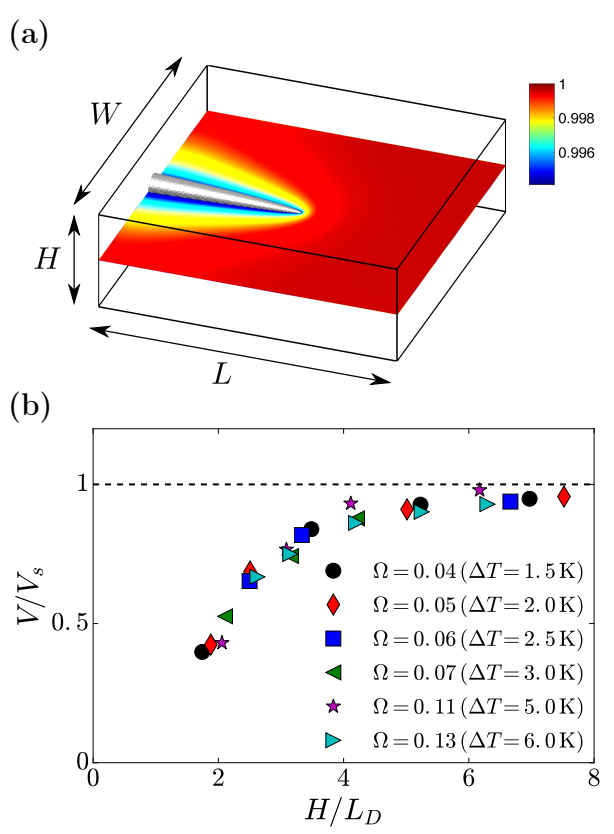

(c)

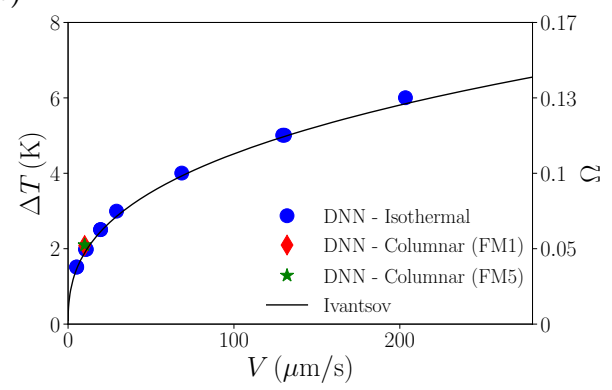

Figure 3: (a) A snapshot of a single DNN dendrite in a slab of thickness $H$ in isothermal conditions. (b) Normalized steady-state velocity as function of simulation height obtained from isothermal DNN simulations for different supersaturation $\Omega$. The dendritic growth velocity is in good agreement with the Ivantsov prediction when $H \geq 4.5 L_{D}$. (c) Steady-state velocity of dendrites obtained for different supersaturation in the case $H \geq 4.5 L_{D}$ (blue circles) compared with the Ivantsov steadystate solution (solid line). The front undercooling obtained from directional solidification simulations representative of FM1 (red diamond) and FM5 (green star) conditions are also reported and show a good agreement with the Ivantsov solution. Parameters used in these simulations can be found in table 2 .

For a steady-state dendrite growth in isothermal condition, the tip radius $\rho_{s}$ and the tip velocity $V_{s}$ are obtained analytically by combining the solvability condition in Eq. (16) and the steady-state Ivantsov relation in Eq. (17). Here the mesh size is set to be $d x=\rho_{s}$, and we investigate the effect of the slab geometry on the dendritic growth kinetics by varying the sample thickness $H$ between 2 and 8 times the diffusion length $L_{D}=D / V_{s}$. 
To reduce the size effect from the other two dimensions, we choose the length and the width of the simulation slab at least 10 times greater than the diffusion length, i.e., $L, W>10 L_{D}$ (see Fig. 3a).

Simulation results in Fig. 3b show the normalized dendritic tip $V / V_{s}$ as a function of $H / L_{D}$ for different supersaturation $\Omega$. Simulation results show that for thick simulation slabs $\left(H / L_{D} \geq 4.5\right)$, the tip velocity converges towards $V_{s}$ and the finite size effect remains limited. Moreover, the results obtained for different supersaturations collapse on a single curve. These two observations indicate that the isothermal DNN model is well implemented and reproduces the appropriate steady-state solution. We plot in Fig. $3 \mathrm{c}$ the undercooling $\Delta T$ as a function of the dendrite tip velocity to show that the DNN method recovers Ivantsov solution.

To test whether the conditions of CETSOL experiments lies within the scope of validity of the DNN model, we performed directional solidification simulations with a pulling velocity $V_{p}=10 \mu \mathrm{m} / \mathrm{s}$ and a thermal gradient $G=1 \mathrm{~K} / \mathrm{mm}$ that corresponds approximately to stage I of the CETSOL experiments (see Section 6 for details). In addition, we plotted the tip undercooling obtained from the simulations for both FM1 and FM5 in Fig. 3c to show a good agreement with the Ivantsov solution, where the front undercooling data are collected at time $t=2000 \mathrm{~s}$, at the end of stage I of the solidification process when the columnar front has reached a steady-state growth regime (cf. Figs. 7a-b). This comparison demonstrates that the CETSOL experiment conditions lie well in the domain of validity of the DNN model.

\subsection{Growth of misoriented dendrites}

In the previous section, we showed that the DNN results reproduce the expected steady-state Ivantsov solution for dendrites aligned with the computational grid. Here we carried out simulations to demonstrate the ability of the DNN approach to model the growth of arbitrarily oriented dendrites as represented in Fig. 1. This is a key ingredient for modeling the CET in this work, which is caused by the blocking of the columnar front by the randomly oriented grains.

Fig. 4 shows the supersaturation as a function of the Péclet number for different needle orientations. The dimension of the simulation domain are $L, H, W \geq 9 L_{D}$ such that the finite size effects remain negligible. Despite a small discrepancy observed for large Péclet numbers, the DNN model is able to reproduce accurately the expected steady-state given by the Ivantsov solution for any needle orientations and for supersaturations in the range of experimental relevance. A similar discrepancy at high Péclet numbers was also obtained in 2D (see Fig. 3 of Ref.[9]) and is attributed to the computation of the FIF from an integration box. To highlight the difference of growth kinetics in 2D and 3D, the Ivantsov solution associated with $2 \mathrm{D}$ dendritic growth is also shown with a dash line.

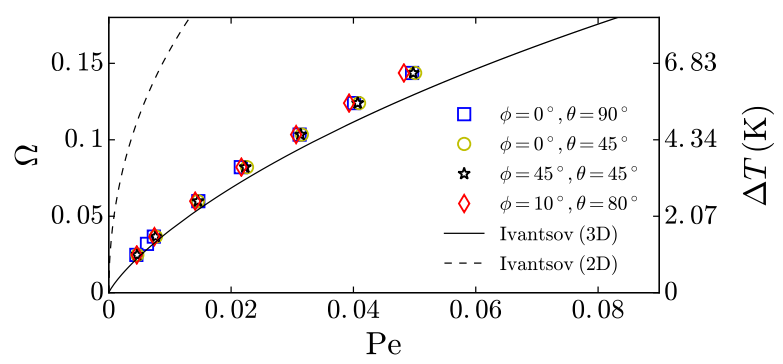

Figure 4: Supersaturation $\Omega$ versus Péclet number Pe obtained for different orientations of the dendritic needle and comparison with the steadystate Ivantsov solution. The right vertical axis represents the undercooling $\Delta T$ related to the supersaturation $\Omega$ by Eq. (27).

\section{Columnar front}

In this section, we extended the study of columnar growth from 2D (see part I [9]) to 3D. The simulations were performed using a standard Bridgman setup, where a sample is pulled at a constant velocity $V_{p}$ in a fixed temperature gradient $G$. The frozen temperature approximation for the thermal field is used: $T(x, t)=T_{0}+G\left(x-V_{p} t\right)$. Those simulations also provide a reasonable approximation of the growth conditions during stage I of solidification in FM1 and FM5 samples for $V_{p}=10 \mu \mathrm{m} / \mathrm{s}$ and $G=1 \mathrm{~K} / \mathrm{mm}$.

In simulations under non-isothermal solidification conditions, it is convenient to rewrite the DNN model equations by measuring length in units of a characteristic length scale $\rho_{c}$. Note that $\rho_{c}$ in simulations is set to be comparable to the steady-state dendrite tip radius $\rho_{s}$ at velocity $V_{p}$, but not exactly equal to $\rho_{s}$ owing to the fact that the dendrite tip radius can evolve in time during the initial transient regime that leads to the establishment of a steady-state columnar front or during changes of pulling velocity in different stages of CETSOL experiments. Unless mentioned otherwise, $\rho_{c}=9.487 \mu \mathrm{m}$ is used in all following non-isothermal solidification simulations. In addition, the mesh size in simulations is chosen to roughly be this characteristic length scale, i.e., $d x \approx \rho_{c}$, and the height of the integral contour box used for FIF calculations is chosen to be $H_{\text {box }} / d x=3-5$.

As discussed in the previous studies $[11,12,13]$ as well as in part I of this paper [9], the side-branching events in 
the DNN model is considered to take place at a distance $d$ behind the tip once the needle advances by this distance. To describe the random spatial fluctuation of sidebranching events, the distance $d$ is randomly distributed in $(N \pm \Delta N / 2) \rho$. We note that when $N$ is comparable to $H_{\text {box }}$, there might be undesired overlaps between integration boxes used to compute the FIF of neighboring dendrites. Here, we choose $N=25$ and $\Delta N=5$ to ensure that dendritic branches are well separated. Furthermore, when the growth velocity of needles approaches zero, the solvability condition in Eq. (16) yields an infinite tip radius, which is nonphysical and leads to possible artifacts. As discussed in the previous work $[11,12,13]$, the thickness of needles is set to be bounded to $R_{\max }=5 \rho_{c}$ to prevent the undesired numerical instabilities.

\begin{tabular}{cccc} 
Symbol & Value & Unit & Ref. \\
\hline$T_{\text {liq }}$ & 618 & ${ }^{\circ} \mathrm{C}$ & {$[14,17]$} \\
$c_{\infty}$ & 7 & wt. $\% \mathrm{Si}$ & {$[14,15,17]$} \\
$k$ & 0.13 & - & {$[29]$} \\
$m_{l}$ & -6.5 & $\mathrm{~K} \cdot \mathrm{wt} \% \mathrm{Si}$ & {$[29]$} \\
$D$ & $3 \times 10^{-9}$ & $\mathrm{~m}^{2} / \mathrm{s}$ & {$[18]$} \\
$\Gamma$ & $1.96 \times 10^{-7}$ & $\mathrm{~K} \cdot \mathrm{m}$ & {$[29]$} \\
$\sigma$ & 0.058 & - & {$[25,26,11,13]$} \\
$d x / \rho_{s}$ & $1-1.5$ & - & \\
$N$ & 25 & - & \\
$\Delta N$ & 5 & - &
\end{tabular}

Table 2: Parameters pertaining to Al-7 wt $\%$ Si alloy used for all simulations in this DNN study.

\subsection{Primary spacing $\Lambda$ : elimination, stability, and branching}

To investigate the columnar competition and the sidebranching instability, we carried out DNN simulations of two neighboring columnar grains growing along the $x$ axis in a simulation domain of length $L$, width $W$ and height $H$ along $x, y$, and $z$ directions, respectively. Figure 5a illustrates the simulation setup, where two parallel needles of length $L / 2$ are initially placed at $y=0$ and $y=W$. We varied the width of the simulation domain $W$ between $0.19 \mathrm{~mm}$ and $4.27 \mathrm{~mm}$ to study the influence of the primary spacing on the tip undercooling and on the side-branching instability. We consider a Bridgman-type setup with a constant thermal gradient $G=1 \mathrm{~K} / \mathrm{mm}$ and a pulling velocity $V_{p}=10 \mu \mathrm{m} / \mathrm{s}$. We impose no-flux boundary condition in the $y$-direction, such that the initial spacing is $\Lambda=W$. The height and length of the system are chosen $H=L=7.69 \mathrm{~mm}$, large enough to avoid finite size effects $\left(H, W>10 L_{D}\right.$, where $\left.L_{D}=300 \mu \mathrm{m}\right)$. (a)

(b)

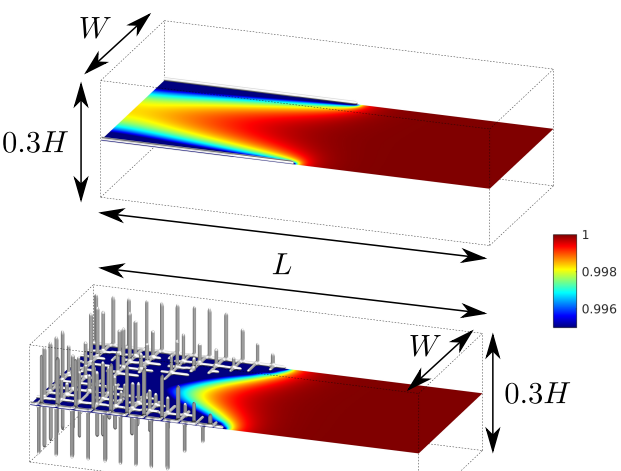

(c)

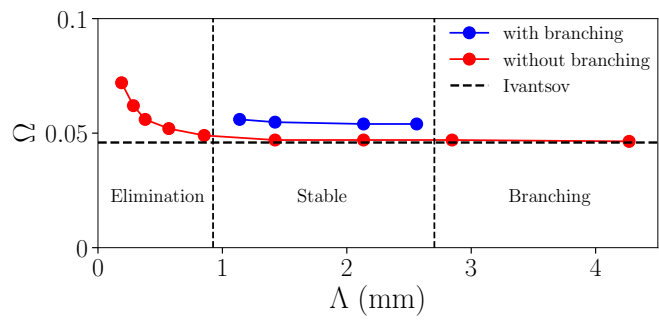

Figure 5: The reduced solute field $u$ between two parallel columnar dendrites in their steady-state (a) without and (b) with secondary branching (only close-up views of the entire simulation slab are shown in panels (a) and (b)). (c) Stability diagram of the columnar front growth as function of the primary spacing $\Lambda$ showing regions of dendrite elimination due solutal interaction, stability and tertiary branching. For these simulations, the parameters listed in Tab. 2 are used together with a pulling velocity $V_{p}=10 \mu \mathrm{m} / \mathrm{s}$ and a thermal gradient $G=1 \mathrm{~K} / \mathrm{mm}$.

To simplify the condition, we suppress the sidebranching of the secondary branches along the $z$ direction to ensure that all $x$-directional dendrites are in the $z=H / 2$ plane. Also, to avoid a nonphysical rapid growth of dendrites along the $z$ direction, these vertical branches are stopped once their length reach a cutoff value $L_{c}=0.75 \mathrm{~mm}$. We checked that the value of this cutoff plays an insignificant role in the branching-stability by varying $L_{c}$ in the range $0.19 \mathrm{~mm}-0.75 \mathrm{~mm}$. Figure $5 \mathrm{~b}$ shows a close-up view of the region between $z=0.35 \mathrm{H}$ and $z=0.65 H$ of the DNN simulated dendritic growth.

We first consider the case of suppressed branching where two parallel needles grow concurrently for any initial spacing $\Lambda$ (see Fig. 5a). Fig. $5 \mathrm{c}$ reports the steadystate tip supersaturation as function of $\Lambda$ with red circles. For spacing $\Lambda>1 \mathrm{~mm}$, the measured tip supersaturation is in very good agreement with the Ivantsov solution (dash line). For $\Lambda<1 \mathrm{~mm}$, the numerical results deviate significantly from the Ivantsov solution due to the solutal interactions between the dendritic tips.

When branching is activated (blue circles in Fig. 5a), 
we obtain three different regimes of primary spacing evolution: elimination, stability, and branching-instability. For an initial spacing $\Lambda<\Lambda_{\min }$, one of the two initial dendrites is overgrown by the other one. This is permitted by the perturbation of the solutal field introduced by branching. For $\Lambda_{\min } \leq \Lambda \leq \Lambda_{\max }$, the spacing is stable (see Fig. 5b). Finally, when $\Lambda>\Lambda_{\max }$, a tertiary branch develops as a new primary columnar dendrite and thereby reduces the initial primary spacing by half. As shown in Fig. 5c, the stability domain stretches between $\Lambda_{\min }=0.92 \mathrm{~mm}$ and $\Lambda_{\max }=3.21 \mathrm{~mm}$. The blue data points in Fig. $5 \mathrm{c}$ show the tip undercooling obtained in the stable regime. The discrepancy between blue and red data points is attributed to the influence of the secondary and tertiary branches on the solutal field that rises the tip supersaturation by approximately $15 \%$. The increase of the undercooling of the columnar front by secondary and tertiary branches is in qualitative agreement with our previous findings reported in part I of this paper [9].

\subsection{Columnar front development}

To complete our study of the columnar front in 3D, we investigate the development of a stable dendritic columnar front. To do so, a regular network of dendrites oriented along the $x$-direction is introduced with an initial spacing $\Lambda_{0}=15 d x-20 d x$ to mimic a planar columnar front (see Fig. 6a).

The initial length of the dendrites is set to $30 d x$, with variations $10 \mathrm{dx}$ in length introduced randomly to accelerate the initial transient elimination of needles before a steady-state columnar front is established. A snapshot of the initial configuration and steady-state configuration are shown in Figs. $6 a$ and $b$.

The area of the cross section in the $y z$ plane is denoted by $A_{y z}=H W$, and the number of surviving primary dendrites is denoted by $N_{\text {surviving. The average spac- }}$ ing of each columnar grain is then simply defined as $\Lambda=\sqrt{A_{y z} / N_{\text {surviving }}}$.

Figs. $6 \mathrm{c}$ and $\mathrm{d}$ show the spacing $\Lambda$ and the supersaturation $\Omega$ of the most advanced tip against the pulling velocity from simulations with temperature gradients $G$ equal to $1 \mathrm{~K} / \mathrm{mm}$ and $5 \mathrm{~K} / \mathrm{mm}$. Our simulation results indicate that the spacing decreases when either the pulling velocity or temperature gradient increase. On the other hand, Fig. 6d shows that the tip supersaturation increases with pulling velocity, while the temperature gradient does not have a significant effect as expected from Ivantsov theory (dash line in Fig. 6d).

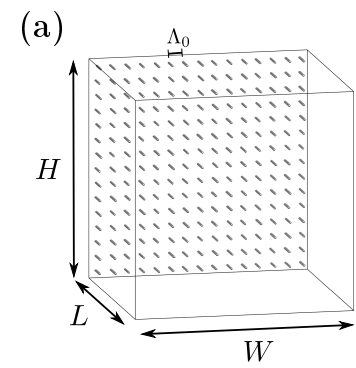

(b)
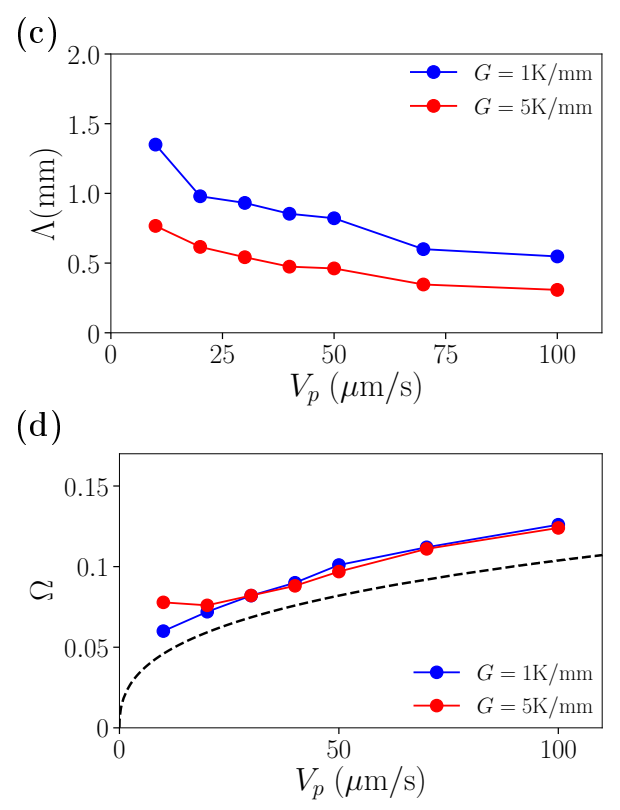

Figure 6: (a) Initial and (b) steady-state morphology of columnar dendrites. (c) Selected primary dendritic spacing from the destabilization of a planar front for different thermal gradient $G$ and pulling velocity $V_{p}$. (d) Supersaturation $\Omega$ as a function of $V_{p}$ for two different values of $G$ and comparison with the Ivantsov solution (dashed line). Here, $\rho_{c}=9.487 \mu \mathrm{m}, d x=1.5 \rho_{c}$ and $L=W=H=300 d x=4269.15 \mu \mathrm{m}$. Here the diffusion length $L_{D}=D / V_{p}$ ranges between $300-30 \mu \mathrm{m}$, so $L, H, W>14 L_{D}$ and therefore the finite size effect can be neglected. Other simulation parameters are listed in Table 2.

The results in Fig. 6d (see also Fig. 5c) reveal that the supersaturation of the columnar front deviates about $20 \%$ from the Ivantsov solution because of the solutal interaction of the primary dendritic tips with the secondary and the tertiary branches. This deviation is comparable to the value found in our 2D study reported in part I of the paper [9].

This section demonstrates that the 3D DNN model enables to investigate quantitatively the properties of the columnar front developed in 3D (tip undercooling, primary spacing) while accounting for the complexity of the columnar front structure and the details of the solutal in- 
teractions.

\section{Nucleation of equiaxed grains}

\subsection{Heterogeneous nucleation}

In CETSOL experiments, $\mathrm{TiB}_{2}$ particles are added in the Al-Si melt to promote the heterogeneous nucleation, which leads to the CET. The nucleation of new grains from inoculant particles is treated in the same way as discussed in part I of the paper [9]. Let us consider a refiner particle at position $\mathbf{x}$ with a background concentration $c(\mathbf{x})$. As nucleation takes place, the background concentration $c(\mathbf{x})$ is deemed as the concentration on the liquid side of the solid-liquid interface $c_{l}(\mathbf{x})$, which is related to the temperature $T(\mathbf{x})$ through Eq. (4). Thus, the onset nucleation temperature in the DNN model is given by

$$
T_{n}=T_{M}+m_{l} c(\mathbf{x})-\Delta T_{n},
$$

where $\Delta T_{n}$ is the nucleation undercooling temperature.

We consider that the refiner particle nucleates once its temperature $T^{\text {refiner }}(\mathbf{x})$ reaches the nucleation temperature $T_{n}$.

The reduced solute field of the refiner particle is defined using Eq. (9) as

$$
u_{l}^{\text {refiner }}(\mathbf{x})=\frac{T_{0}-T^{\text {refiner }}(\mathbf{x})}{T_{0}-T_{L}} .
$$

Combining Eqs. (28) and (29), we obtain the following condition on the local solutal field:

$$
u(\mathbf{x})=u_{l}^{\text {refiner }}+\Delta u_{n}
$$

where the undercooling concentration is defined as

$$
\Delta u_{n} \equiv \frac{\Delta T_{n}}{\left|m_{l}\right| c_{l}^{0}(1-k)} .
$$

\subsection{Development of new grains from nucleant particles}

The DNN model requires a finite contour box for the FIF computation for each dendrite. Therefore, the contour integral becomes unachievable when the dendrite length is smaller than a reasonable size of contour box, making difficult to integrate the growth of grains just after their nucleation. To resolve this issue, we consider that a nucleated particle first develops as a spherical germ. The growth of the spherical germ is treated in the same way as discussed in part I of the paper[9]: its growth rate is obtained from the incoming flux of solute computed by the means of a cubic integration box containing the precipitate. When the germ radius reaches a threshold value $R_{c}=4 \rho_{c}$, the spherical particle is replaced by an equiaxed six-arm grain of the same volume. Each arm is then considered as an independent dendrite and is treated with the DNN model. Our previous study in 2D suggests that the specific choice of the destabilization radius $R_{c}$ has a limited influence on the grain evolution except for the early transient growth [9].

\section{Columnar-to-equiaxed transition (CET)}

In Section 4, we have shown that the 3D DNN model is able to describe accurately the columnar front by accounting for the details of the grain structure and the dendritic solutal interactions. Moreover, in Section 5 the model has been extended to account for the heterogeneous nucleation on inoculant particles and the growth of equiaxed grains. In this section, the 3D DNN model is used to study the CET by imposing the thermal conditions obtained from the CETSOL experiments.

\subsection{CET experiments}

Directional solidification experiments of Al-7 wt.\%Si alloys were conducted onboard the International Space Station in the framework of the CETSOL project. The main goal of these experiments is to study the columnar-to-equiaxed transition in microgravity, where the sedimentation and the buoyancy-driven flow are suppressed $[14,15,17]$. In these flight experiments, cylindrical bulk samples with a diameter of $7.8 \mathrm{~mm}$ and a length of $245 \mathrm{~mm}$ are inserted in the sample cartridge assembly. The directional solidification is achieved by a cooling process consisting of three consecutive solidification stages, denoted by stages I, II and III. Experiments with and without inoculant particles for different cooling schemes were performed to investigate the influences of solidification velocity, temperature gradient, inoculant concentration on the CET. These experimental studies suggest that the presence of inoculants substantially enhances the CET by promoting the nucleation of new equiaxed grains ahead of the columnar front [14, 15, 17].

Here, we focus on the CET in FM1 and FM5 experiments with inoculant $\mathrm{TiB}_{2}$ particles. The solidification procedure for these two experiments are described below. The desired temperature gradient along the solidification direction is achieved by controlling independently seven heaters. Each individual sample undergoes three consecutive stages (I, II, III) of cooling before the solidification is completed.

- In stage I, directional solidification is initiated by pulling the furnace at a velocity from zero to $V_{p}=$ $0.01 \mathrm{~mm} / \mathrm{s}$ while the temperature gradient is kept at $0.9 \mathrm{~K} / \mathrm{mm}$ for 2000 seconds. 
- During stage II, along with cooling-down of the heaters in the hot zone at a rate of $0.067 \mathrm{~K} / \mathrm{s}$, the pulling velocity in FM1 jumps to a much higher value, $V_{p}=0.2 \mathrm{~mm} / \mathrm{s}$, for 250 seconds, while the pulling velocity in FM5 remains unchanged $\left(V_{p}=\right.$ $0.01 \mathrm{~mm} / \mathrm{s}$ ) for the next $2000 \mathrm{sec}$.

- During stage III, the solidification in both FM1 and FM5 experiments is accelerated by switching off all the heaters and applying a rapid movement of the furnace at a high pulling velocity $V_{p}=3 \mathrm{~mm} / \mathrm{s}$.
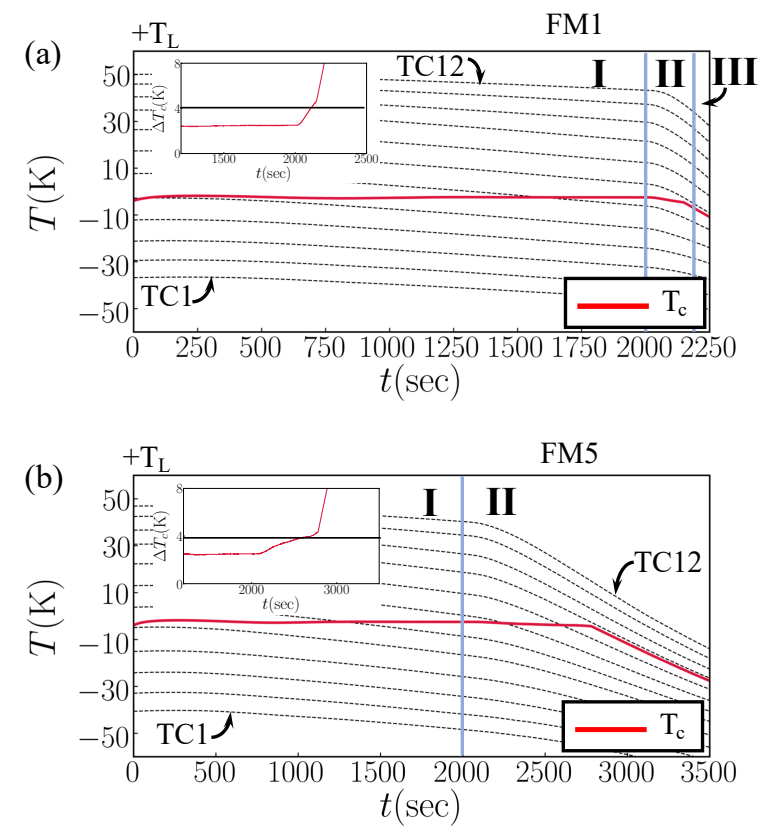

Figure 7: Temperatures recorded from 12 thermocouples (TC1 - TC12, dashed lines) is both (a) FM1 and (b) FM5 samples and the temperature of the columnar front $T_{c}$ (red lines) obtained in our simulations. The insets of (a) and (b) display the time evolution of the columnar front undercooling $\Delta T_{c}$ and compares it to the nucleation undercooling $\Delta T_{n}=$ $4 \mathrm{~K}$ used in simulations.

The temperature profiles in the samples during the solidification procedure are collected by 12 thermocouples (denoted TC1 to TC12), which are located at positions from $72.5 \mathrm{~mm}$ (TC1) to $182.5 \mathrm{~mm}$ (TC12) with an interval of $10 \mathrm{~mm}$. Fig. 7a and b display the time evolution of the temperature profiles in FM1 and FM5 samples, respectively $[14,15,17]$. The red lines represent the columnar front undercooling obtained in our DNN simulation (see Section 6.2 for details). We note that in contrast with simulations performed with a thin slab geometry reported in Ref. [16], the red lines in Fig. 7a are obtained from a full 3D simulation. The insets of Fig. $7 \mathrm{a}$ and $\mathrm{b}$ show the time evolution of the columnar front undercooling (in red) compared with the nucleation undercooling of the inoculant particles $\Delta T_{n}=4 \mathrm{~K}$ (black line) used in our simulations. It is expected that the CET takes place when the columnar front undercooling exceeds the nucleation undercooling, allowing columnar grain to nucleate ahead of the columnar front. In practice, it was found that the CET takes place at stage II in both FM1 and FM5 experiments.

\subsection{DNN CET simulations}

In order to study the CET, inoculant particles are introduced in our simulations to promote the nucleation of grains ahead of the columnar front. As suggested by the experimental studies [14, 15], we choose the nucleation undercooling temperature $\Delta T_{n}=4 \mathrm{~K}$ and the effective density of inoculant $\rho_{n}=1.25 / \mathrm{mm}^{3}$.

Instead of using the frozen temperature approximation of a Bridgman setup, where the thermal condition is controlled by a thermal gradient and a pulling velocity, we directly impose the experimental temperature profile as the input of simulations for the thermal condition.

The initial condition for columnar growth in simulations is obtained by inserting an array of parallel columnar dendrites with primary dendritic spacing $\Lambda_{\text {ini }}=$ $0.6 \mathrm{~mm}$ [13] and initial columnar front undercooling $\Delta T_{c}^{\text {ini }}=4 \mathrm{~K}$. We also performed simulations by varying $\Delta T_{c}^{\text {ini }}$ between $2-8 \mathrm{~K}$ to show that the model with different initial undercooling produced the same columnar growth except for the early transient growth regime (less than $\sim 1000 \mathrm{~s}$ ).

The development of higher order side-branching is not restricted in our simulations, but the results suggest that allowing up to the tertiary branching is enough to reproduce accurately the columnar growth. The temperature of the most advanced columnar tip $T_{c}$, is monitored during the solidification process and shown in Fig. 7a and $b$.

It is computationally expensive to model the entire CETSOL samples with the DNN approach. According to the study of the finite size effect in Section 3, the 3D growth kinetics of branches is fully reproduced in the DNN model as long as the thickness of the slab is large enough, i.e., $H / L_{D} \geq 4.5$. We obtain the maximum diffusion length $L_{D}=D / V_{p} \approx 0.3 \mathrm{~mm}$ of the CETSOL experiments by using the lowest pulling velocity in experiments $V=0.01 \mathrm{~mm} / \mathrm{s}$. We set our simulation box sizes to $L \times W \times H=15.2 \mathrm{~mm} \times 5.5 \mathrm{~mm} \times 2.3 \mathrm{~mm}$ which is thin enough to be computationally tractable but thick enough compared to $L_{D}$ to reduce the finite size effect. Other simulation parameters are shown in Table 3. 


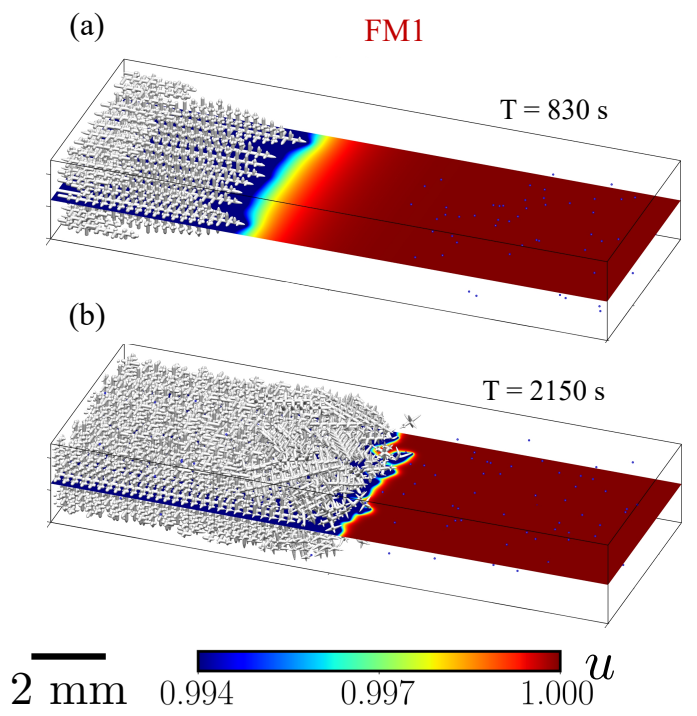

Figure 8: Snapshots at (a) $t=830 \mathrm{~s}$ and (b) $t=2150 \mathrm{~s}$ of DNN simulation results for thermal conditions of FM1. The sample contains $\mathrm{TiB}_{2}$ inoculants with undercooling $\Delta \mathrm{T}_{\mathrm{n}}=4 \mathrm{~K}$ and density $\rho_{\mathrm{n}}=1.25 / \mathrm{mm}^{3}$. (a) In stage I, the directional solidification process is realized by pulling the sample at a velocity from zero to $V_{p}=0.01 \mathrm{~mm} / \mathrm{s}$ with a constant temperature gradient $G=0.9 \mathrm{~K} / \mathrm{mm}$ for 2000 seconds. At $t=2000 \mathrm{~s}$, stage II starts and the pulling velocity jumps to $V_{p}=0.2 \mathrm{~mm} / \mathrm{s}$. (b) At $t=2150 \mathrm{~s}$, the columnar front is completely blocked by equiaxed growth of nucleants. In all panels, blue dots in front of the columnar grains represent inoculant particles.

Figures 8 and 9 display the snapshots of the DNN simulated dendrites in FM1 and FM5 samples. Since the solidification schemes at stage $\mathrm{I}(t=0-2000 \mathrm{~s})$ in both FM1 and FM5 are identical, the simulation results, as expected, show the same dynamics of dendritic growth in both samples (see Figs. 8a and 9a for FM1 and FM5, respectively). In stage II (starting at $t=2000 \mathrm{~s}$ ), the pulling velocity of FM5 sample remains $V_{p}=0.01 \mathrm{~mm} / \mathrm{s}$ for another 2000 seconds while the pulling velocity of FM1 sample is increased 20-fold to $V_{p}=0.2 \mathrm{~mm} / \mathrm{s}$ for 250 seconds. As in the experiments, our simulations show that the CET occurs during stage II for both FM1 and FM5 samples. Furthermore, we observe a much sharper CET in FM1 compared to FM5 due to the fast cooling process of FM1: at $t=2150 \mathrm{~s}$, all the columnar dendrites in FM1 are already blocked by equiaxed grains (see Fig 8b) while in FM5, the columnar front is still growing freely (see Fig. 9b). The nucleation and the development of equiaxed grains in FM5 occurred at a much later time. The snapshot in Fig. 9c shows that at $t=2700 \mathrm{~s}$, all columnar dendrites but one (see the inset) are blocked by the growth of equiaxed grains ahead of them. Finally, as shown in Fig. 9d, at

$t=2790 \mathrm{~s}$ the columnar front is fully blocked by equiaxed grains.

(a)
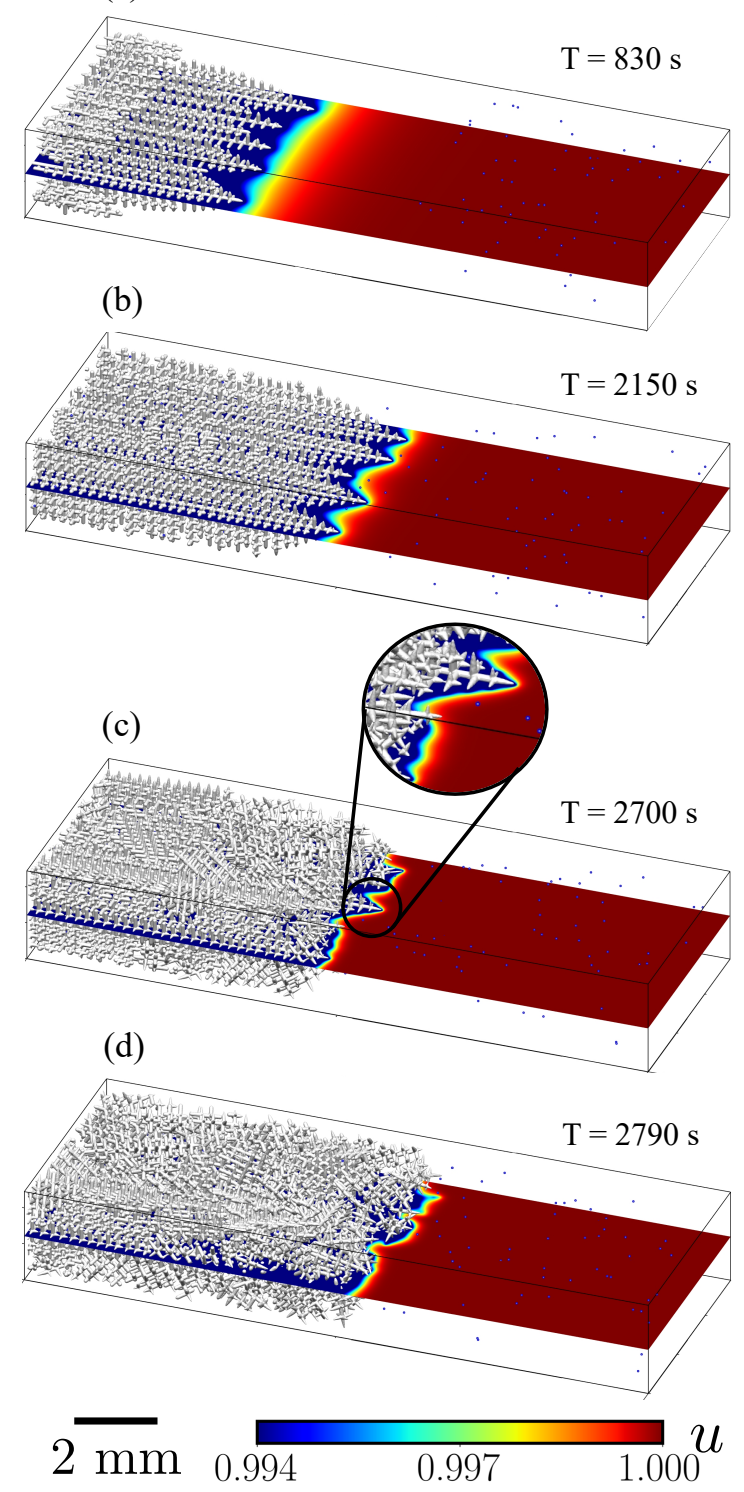

Figure 9: Snapshots at (a) $t=830 \mathrm{~s}$, (b) $t=2150 \mathrm{~s}$, (c) $t=2700 \mathrm{~s}$ and (d) $t=2790 \mathrm{~s}$ of DNN simulation results for thermal conditions of the sample FM5. TiB 2 inoculants with undercooling $\Delta \mathrm{T}_{\mathrm{n}}=4 \mathrm{~K}$ and an effective density $\rho_{\mathrm{n}}=1.25 / \mathrm{mm}^{3}$. (a) Stage I of the solidification process (pulling velocity $V_{p}=0.01 \mathrm{~mm} / \mathrm{s}$ ) with the development of the columnar front. In stage II of FM5, the pulling velocity remains $V_{p}=0.01 \mathrm{~mm} / \mathrm{s}$. At $t=2150 \mathrm{~s}(\mathrm{~b})$, nucleation does not occur ahead of the columnar front. The only surviving columnar dendrite observed at $t=2700 \mathrm{~s}$ in (c) is blocked at $t=2790 \mathrm{~s}$ in (d). In all panels, blue dots in front of the columnar grains represent inoculant particles.

The CET starting point $\mathrm{CET}_{\min }$ can be defined as the 


\begin{tabular}{ccc} 
Symbol & Value & Unit \\
\hline$\rho_{c}$ & $9.487 \times 10^{-6}$ & $\mathrm{~m}$ \\
$\tau_{c}$ & 1.722 & $\mathrm{~s}$ \\
$d t$ & $4.5 \times 10^{-3}$ & $\mathrm{~s}$ \\
$d x / \rho_{c}$ & 1 & - \\
$N$ & 25 & - \\
$\Delta N$ & 5 & -
\end{tabular}

Table 3: Simulation parameters used in simulations DNN-FM1 and DNN-FM5.

place where the first inoculant particle starts to develop as an equiaxed grain. On the other hand, different methods can be used to locate the ending point of the transition denoted $\mathrm{CET}_{\max }$. One way is to use the location of the most advanced columnar grain [16]. Another method consist in considering the average grain elongation denoted $\gamma$ to identify $\mathrm{CET}_{\max }$ : where $\gamma$ drops below 2 can be marked as the CET ending point $[15,16]$. In our simulations, the maximum position $\mathrm{CET}_{\max }$ is measured using both of these methods, whichever yields the larger value. Finally, the transition length is defined as $\ell_{\mathrm{CET}}=$ $\mathrm{CET}_{\text {max }}-\mathrm{CET}_{\text {min }}$. The values of $\mathrm{CET}_{\text {max }}, \mathrm{CET}_{\text {min }}$ and $\ell_{\text {CET }}$ obtained numerically and experimentally are compared in Tab. 4. We highlight the quantitative nature of the DNN results that accurately predict the start, the end and the length of the CET without any adjustable parameter.

\begin{tabular}{cc|c|c} 
& & CETSOL & DNN \\
\hline \multirow{3}{*}{ FM1 } & CET $_{\text {min }}$ & $125 \mathrm{~mm}$ & $127 \mathrm{~mm}$ \\
& $\mathrm{CET}_{\text {max }}$ & $130 \mathrm{~mm}$ & $130 \mathrm{~mm}$ \\
& $\ell_{\text {CET }}$ & $5 \mathrm{~mm}$ & $3 \mathrm{~mm}$ \\
\hline \multirow{3}{*}{ FM5 } & $\mathrm{CET}_{\min }$ & $130 \mathrm{~mm}$ & $134 \mathrm{~mm}$ \\
& $\mathrm{CET}_{\max }$ & $156 \mathrm{~mm}$ & $156 \mathrm{~mm}$ \\
& $\ell_{\text {CET }}$ & $26 \mathrm{~mm}$ & $22 \mathrm{~mm}$
\end{tabular}

Table 4: Quantitative comparison bewteen DNN prediction and CETSOL experimental results for FM1 and FM5 samples [15, 17, 14]

To better understand the CET, we analyze more precisely the structure of the equiaxed grains in our DNN simulations. To do so, we consider three characteristics of the grains: the grain size $d_{g}$, the elongation factor $\gamma$, and the grain orientation with respect to the pulling direction $\alpha_{m}$. A schematic of a 3D equiaxed gain is shown in Fig. 10. Each grain has three sets of paired equiaxed arms with length $d_{i j}$ with the corresponding growth direction $\hat{t}_{i j}$. Index $i=1,2,3$ represents each set of paired arms and index $j=1,2$ corresponds to the first or second arm of each set, i.e. $\hat{t}_{i 1}=-\hat{t}_{i 2}$. The total length of each set of paired arms can be defined as: $L_{i}=d_{i 1}+d_{i 2}$. We chose the set of $i$ index in such a way that $L_{1} \geq L_{2} \geq L_{3}$. Finally, we define the growth angle of each arm $\alpha_{i j}$ and the grain orientation $\alpha_{m}$ as follow:

$$
\begin{aligned}
& \alpha_{i j}=\arccos \left(\hat{t}_{i j} \cdot \hat{x}\right), \\
& \alpha_{m}=\min \left(\alpha_{11}, \pi-\alpha_{11}\right) .
\end{aligned}
$$

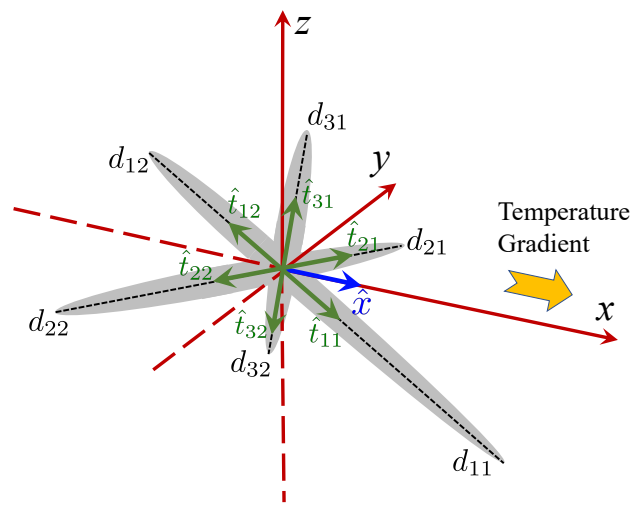

Figure 10: Schematic of an equiaxed grain with six arms, which are pairwise coaxial. We choose set index $i=1,2,3$ to be the set index for the longest, second longest, and the shortest arms, respectively.

Figs. 11 and 12 present the elongation factor, the grain size, and the grain orientation of the nucleated grains as function of position in FM1 and FM5 DNN simulations. These data are color-coded according to the grain orientation angle $\alpha_{m}$ (red and blue tints represent respectively the grains well oriented and misoriented with the thermal gradient). The black lines show the averaged values calculated within intervals of $2 \mathrm{~mm}$. As expected, both elongation and grain size decrease with the position in the CET region, which translates the nucleation of smaller more equiaxed grains ahead of the columnar front.

More precisely, in the sharply transitioned FM1 simulation, the elongation factor drops immediately below 2 (with a transition length of $3 \mathrm{~mm}$ ) while in FM5 simulation, it decreases significantly but remains above 2 over a distance of $22 \mathrm{~mm}$.

In Figs. 11 and Fig. 12, the position of the most advanced columnar tip in FM1 and FM5 simulations is found be to $130 \mathrm{~mm}$ and $150 \mathrm{~mm}$ in FM5 sample, respectively (red vertical dashed lines). Figures 11c and Fig. 12c represent the plots of the grain orientation as a function of the grain position. 


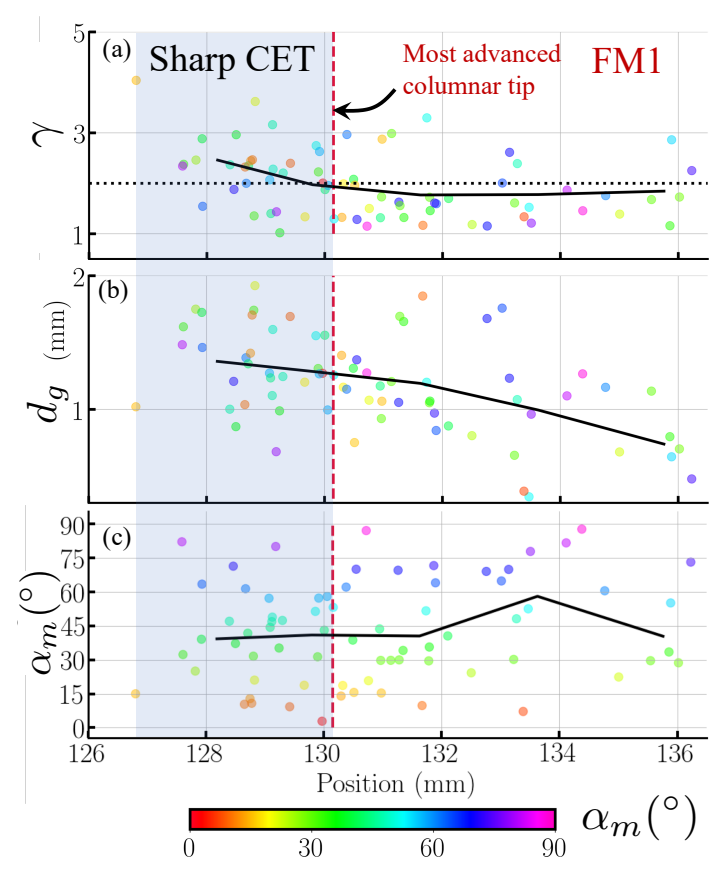

Figure 11: (a) Elongation factor, (b) grain size, and (c) grain orientation of the equiaxed grains as a function of their position for the FM1 simulation. The first nucleation event occurs at the position $127 \mathrm{~mm}$. The vertical red line indicates the stopping point of the last columnar grain and the light blue region represents where the CET takes place. The color map shows the angle between the largest arm of the grain and the temperature gradient axis.

One can see that in FM1 simulation, because the transition length is very short and the initial orientations of grains are assigned randomly, the data is very scattered and the average angle is around $45^{\circ}$, as shown in Fig. 11. However, in FM5 simulation, because the CET length is longer and the columnar and equiaxed grains coexist for a longer time, the arms parallel to the pulling direction are more likely to survive. Therefore, we see that the average angle is less than $45^{\circ}$ at the beginning of the CET and increases as more equiaxed grains grow in front of the columnar front. At the beginning of the CET, Fig. 12 shows that three well-oriented grains with $\alpha_{m}<10^{\circ}$ have elongation factor $\gamma>7$ and grain size $d_{g}>2.5 \mathrm{~mm}$. It shows that nucleated grains with small grain orientation can become new columnar grains.

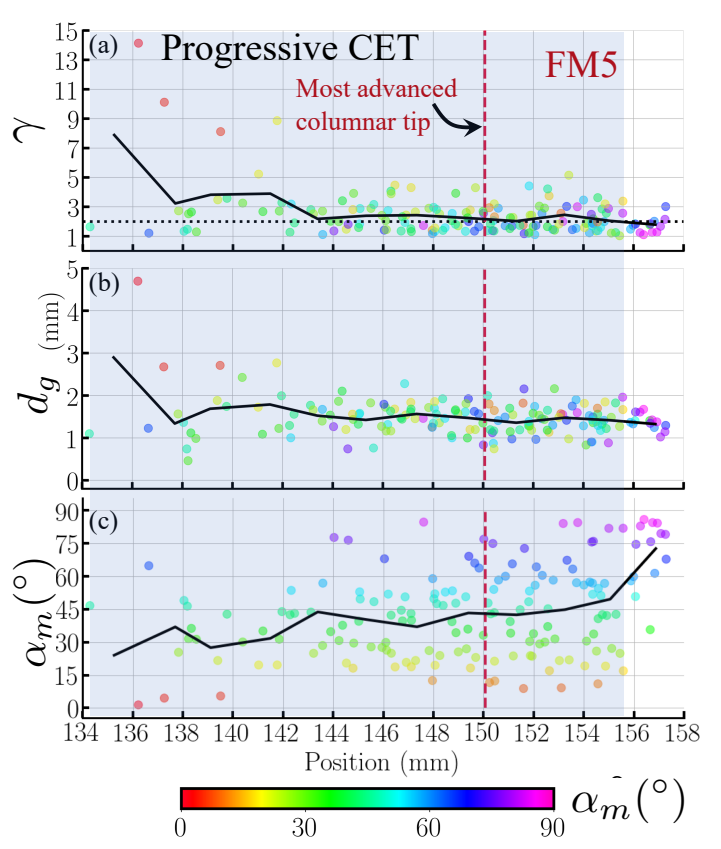

Figure 12: (a) Elongation factor, (b) grain size, and (c) grain orientation as a function of position in FM5 experiment. The first nucleation event occurs at the position $127 \mathrm{~mm}$. The vertical red line indicates the stopping point of the last columnar grain and the light blue region represents where the CET takes place. Panel (a) shows that the elongation factor drops below 2 at $156 \mathrm{~mm}$, marking the end of the CET whose extent is represented with a light blue coloring.

Figure 11 shows the average grain diameter in FM1 simulation quickly drops after the nucleation of the first equiaxed grain and stabilizes around $1 \mathrm{~mm}$ while the average elongation factor drops below 2 on the same lengthscale. On the other hand, Fig. 12 show that in FM5 simulation the grain diameter and the average elongation factor remains around $1.5 \mathrm{~mm}$ and above 2 , respectively, long after the first nucleation event.

Overall, our simulation results suggest two distinct types of CET: sharp CET in FM1 and progressive CET in FM5. In addition, the grain size and elongation factor obtained from simulations are in quantitative agreement with experimental observations [17]. 


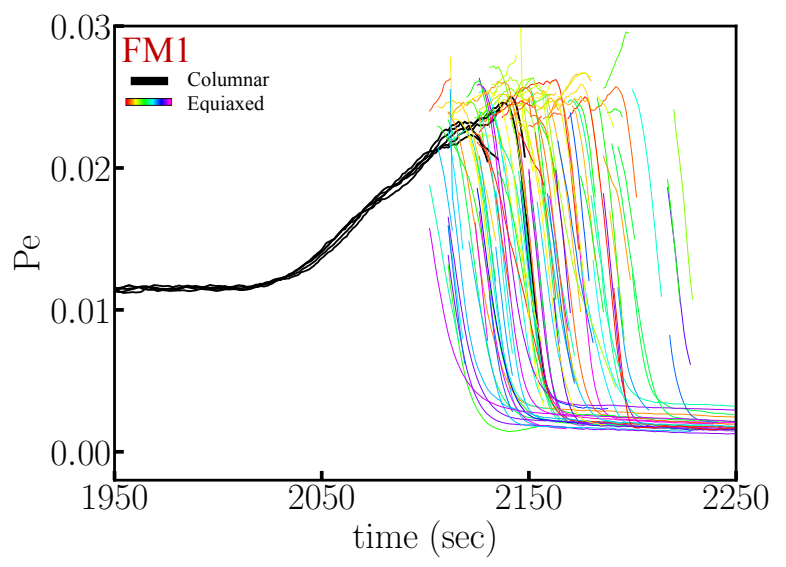

Figure 13: Péclet number of each needle as function of time for FM1 simulation during $1950<t<2250$ seconds. The color map shows the growth direction of each needle with respect to the temperature gradient axis.

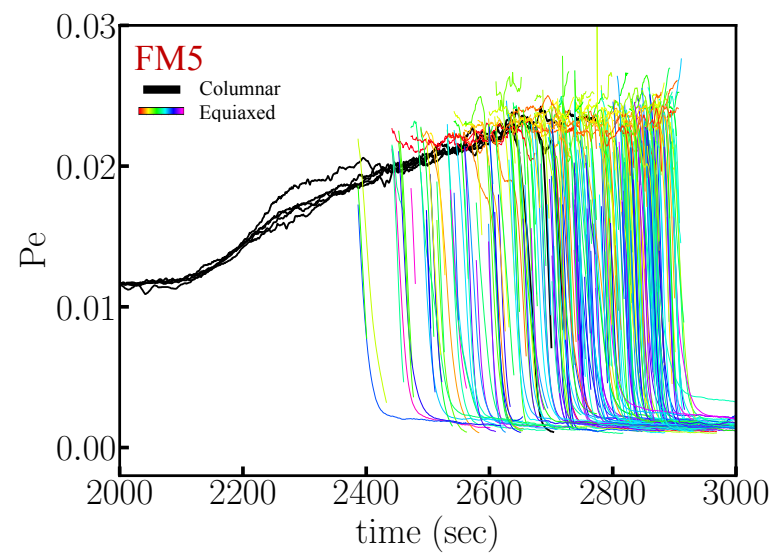

Figure 14: (Péclet number of each needle with respect to time for FM5 sample during $2000<t<3000$ seconds. The color map shows the growth direction of each needle with respect to the temperature gradient axis.

We present in Figs. 13 and 14 the time evolution of the Péclet number of the columnar dendrites and each primary arm of the equiaxed grains during $t=1950-2250 \mathrm{~s}$ for FM1 and $t=2000-3000 \mathrm{~s}$ for FM5, respectively. The data for columnar grains are plotted in black lines when the data for individual dendrites of the nucleated grains are showed in lines color-coded according to their growth angle $\alpha_{i j}$, which is the angle between the growth direction and the pulling direction (see Eq. (32)).

These figures demonstrate that the CET is much more progressive in FM5 than in FM1 simulation: the columnar front continues to grow for another 300 seconds after the first nucleation event in FM5 while in FM1 it is almost immediately blocked. Besides, we found in both Figs. 13 and 14 that the dendrites growing parallel to the temperature gradient direction (red lines) generally survive over a longer time period than the misoriented dendrites (blue lines).

\section{Conclusion}

In this part II, we presented a 3D DNN model and its application to investigate the columnar-to-equiaxed transition (CET) and compare our results with CETSOL experiments. The dendritic tip in the DNN model is described as a paraboloid, and the evolution of the tip radius and the tip velocity are uniquely determined by solving a solvability condition and a mass conservation condition. The former condition is applied at the scale of the tip radius and relates the product $\rho^{2} V_{\text {tip }}$ to the strength of the surface tension anisotropy. The latter condition measures the solute flux directed towards the dendritic tip in terms of a flux intensity factor $\mathcal{F}$, which is computed by a surface integration around the dendrite tip at an intermediate scale between the tip radius and the diffusion length. Hence, the DNN model bridges the dendritic tip scale and grain scale by tracking the dynamics of dendritic networks on the scale of several grains. For this reason, it becomes a powerful numerical approach for modeling the CET.

We discussed in Section 2 the implementation of the DNN model to compute the flux intensity factor for arbitrarily oriented dendrites in 3D. We showed in Section 3 the validation of the model against the Ivantsov solution for the stead-state growth of dendrites of different orientations. In a study of the finite size effect on the dynamics of the dendrites in a slab geometry, we found that the finite size effect becomes insignificant once the sample thickness is 4.5 times greater than the diffusion length. This observation allows us to investigate the CET in a slab geometry which is thin enough to be computationally tractable but at the still thick enough to have negligible finite size effect.

We carried out simulations to study the columnar grain growth and the growth competition. The results in Section 4 demonstrate that the columnar front undercooling observed in simulations is higher than the Ivantsov steadystate solution due to the solutal interactions between dendrites. We found that the deviation of the columnar front undercooling from the Ivantsov steady-state solution in $3 \mathrm{D}$ is comparable to the value obtained from our 2D study reported in part I of this paper [9].

We then applied the 3D DNN model to investigate the CET and the results are used to establish benchmark comparisons with the CET experiments in $\mathrm{Al}-7 \mathrm{wt} \% \mathrm{Si}$ alloy 
samples solidified aboard the ISS under different thermal conditions. The implementation of the model for the nucleation of new grains from inoculant particles is treated in the same way as discussed in part I [9].

By directly imposing the experimental temperature profile as the input of simulations for the thermal condition, the DNN model predicts either a sharp CET in FM1 sample solidified with a sudden increase in the solidification velocity or a progressive CET in FM5 sample solidified with a continuous decrease of the temperature gradient. Our simulation results demonstrate that the present model is also able to predict the start and end positions of the transition in quantitative agreement with experimental results.

In summary, together with previous studies [11, 12, 13], this work shows that the DNN approach is able to well describe the complex dendritic microstructure in 3D during the solidification process. The next step will consist in applying the DNN approach to model quantitatively the $\mathrm{CET}$ in other refined and non-refined $\mathrm{Al}-7 \mathrm{wt} \% \mathrm{Si}$ samples processed under different conditions aboard the ISS. Simulation results will be compared to the detailed measurements of the grain size and grain orientation in the samples using the serial sectioning technique. Furthermore, the DNN model will be used to model the growth of equiaxed dendrites solidified in microgravity in the transparent model alloy Neopentylglycol-DCamphor (NPGDC) during the sounding rocket mission MASER-13.

The DNN model presented in this part is designed to study the CET in microgravity environment where the influence of the gravity-induced melt convection is neglected.

We note that the DNN model has been recently extended to incorporate the role of fluid flow [30], paving the way to investigate the microstructure development with convection flow encountered in solidification experiments under terrestrial conditions. We expect that the incorporation of convection flow in DNN model will enable to improve our understanding of the influence of the heat and solute transport in the melt on several important phenomena in solidification, such as grain nucleation, grain growth, growth competition and dendrite fragmentation in presence of convection.

\section{Acknowledgment}

This research was performed within the framework of the Columnar-to-Equiaxed Transition in Solidification (CETSOL) project funded jointly by NASA and ESA under trant No.80NSSC19K0135. We wish to thank members of this international collaboration, Ch.-A. Gandin,
L. Sturz, and G. Zimmermann for providing us with the thermocouple measurements used in the modeling and for valuable exchanges. The authors also thank Damien Tourret for his contribution to the initial development of the DNN simulation code and his help and remarks during the implementation of the model.

\section{Appendix A. Integration box geometry}

In the present model, an integration box $\Sigma$ is used for computing the FIF of each dendrite. The six sides of the integration box are denoted by $S_{x}^{+}, S_{x}^{-}, S_{y}^{+}, S_{y}^{-}, S_{z}^{+}$, $S_{z}^{+}$corresponding to the left, right, back, front, top, and bottom sides of $\Sigma$ (see Fig. 2a). We consider that each dendrite always passes through one side of the box and this intersected side is denoted by $S$ with the unit normal vector $n_{s}$ directed inward $\Sigma$. The intersected plane $S$ is chosen according to the rule summarized in Table 1 . The paraboloid tip is at $\vec{r}_{\text {tip }}=\left(x_{\text {tip }}, y_{\text {tip }}, z_{\text {tip }}\right)$. The thickness direction of the integration box is chosen as the unit normal vector of the intersected plane, $\hat{n}_{s}$. The tip is kept at the center of the integration box $\Sigma$ along the $\hat{n}_{s}$ direction. In order to ensure that the dendrite intersects with only one side of $\Sigma$, the length and the width of $\Sigma$ need to be dynamically adjusted during the dendrite growth. The approach of determining the geometry of $\Sigma$ is described below.

Without loss of generality, let's first take a C1 needle (see Table 1) as an example, where $\hat{n}_{s}=\hat{z}$ and $S: z-z_{\text {tip }}=$ $-H_{b o x}$ (see Fig. 2a). The paraboloid tip is initially placed at the center of a cuboid integration box of height $2 \mathrm{H}_{\mathrm{box}}$, width $2 W_{\text {box }}$ and length $2 L_{\text {box }}$, as shown in Fig. 2a. The size of the integration box is carefully chosen to ensure that the bottom of the box is the only side intersecting with the dendrite at all times during dendrite growth.

The intersected region of the dendrite and $S$ is an ellipse, which is denoted by

$$
\Gamma_{s}:\left\{\vec{r}_{s}=\left(x_{s}, y_{s},-H_{\mathrm{box}}\right)\right\},
$$

where the intersected region $\Gamma_{s}$ is computed numerically in the code.

$$
\Sigma:\left(x_{\text {box }}^{-}, x_{\text {box }}^{+}\right) \times\left(y_{\text {box }}^{-}, y_{\text {box }}^{+}\right) \times\left(z_{\text {box }}^{-}, z_{\text {box }}^{+}\right)
$$

Where,

$$
\begin{aligned}
& x_{\text {box }}^{+}=\max \left(x_{\text {tip }}+H_{\text {box }},\left\{x_{s}\right\}\right)+\delta_{\text {buffer }} \\
& x_{\text {box }}^{-}=\min \left(x_{\text {tip }}-H_{\text {box }},\left\{x_{s}\right\}\right)-\delta_{\text {buffer }} \\
& y_{\text {box }}^{+}=\max \left(y_{\text {tip }}+H_{\text {box }},\left\{y_{s}\right\}\right)+\delta_{\text {buffer }} \\
& y_{\text {box }}^{-}=\min \left(y_{\text {tip }}-H_{\text {box }},\left\{y_{s}\right\}\right)-\delta_{\text {buffer }} \\
& z_{\text {box }}^{ \pm}=z_{\text {tip }} \pm H_{\text {box }}
\end{aligned}
$$


The model is discretized spatially on a finite difference cubic grid of uniform mesh size $d x$. Here the buffer $\delta_{\text {buffer }}=d x$ is used in simulations to ensure that $\Sigma$ meets the requirement that $S$ is the only side intersecting with the dendrite. The approach mentioned above for $\mathrm{C}-1$ dendrite can be extended for dendrites of other five categories, C2 - C6.

\section{Appendix B. Calculation of the intersection area $\mathcal{A}_{s}$}

To calculate the area of the intersected region $\Sigma$, let's consider that a tilted dendritic paraboloid of growing direction $\hat{t}$ intersects at an angle $\alpha$ with a plane of normal direction $\hat{n}_{s}$. We then choose a Cartesian coordinate $(\xi, \eta, \zeta)$, where $\hat{\zeta}=\hat{n}_{s}, \eta \equiv \hat{\zeta} \times \hat{t}$ and $\hat{\xi} \equiv \hat{\eta} \times \hat{\zeta}$, as seen in Fig. B. 15 . The plane $S$ is given by

$$
S: \zeta=-H_{\mathrm{box}}
$$

the angle $\alpha=\cos ^{-1}(\hat{t} \cdot \hat{\zeta})$, and the tip is located at the origin in this coordinate system.

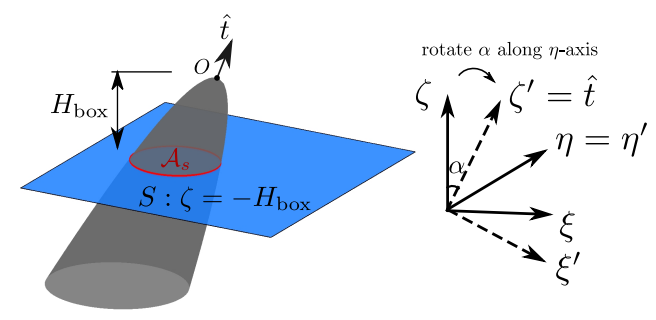

Figure B.15: Illustration showing the intersection $\Gamma_{s}$ between a tilted paraboloid with tip radius $\rho$ and plane $S$. The intersected region $\Gamma_{s}$ is elliptical and the area $\mathcal{A}_{s}=\pi a b$, where $a=W / \cos \alpha$ and $b=W$ are the major radius and minor radius of the ellipse, respectively. $W=$ $\sqrt{(\rho \tan \alpha)^{2}+\frac{2 \rho H_{\text {box }}}{\cos \alpha}}, \alpha$ is the angle between $\hat{\zeta}$ and $\hat{t}$, i.e., $\alpha=\cos ^{-1}(\hat{\zeta} \cdot \hat{t})$.

We consider another coordinate system $\left(\xi^{\prime}, \eta^{\prime}, \zeta^{\prime}\right)$, which is obtained by rotating $(\xi, \eta, \zeta)$ by an angle of $\alpha$ along the $\eta$-axis. Then the two coordinates systems are related through the following relation:

$$
\left(\xi^{\prime}, \eta^{\prime}, \zeta^{\prime}\right)=R_{\alpha}\left(\begin{array}{l}
\xi \\
\eta \\
\zeta
\end{array}\right)
$$

where the rotation matrix is

$$
R_{\alpha}=\left(\begin{array}{ccc}
\cos \alpha & 0 & -\sin \alpha \\
0 & 1 & 0 \\
\sin \alpha & 0 & \cos \alpha
\end{array}\right)
$$

Hence,

$$
\begin{aligned}
& \xi^{\prime}=\xi \cos \alpha-\zeta \sin \alpha \\
& \zeta^{\prime}=\xi \sin \alpha+\zeta \cos \alpha
\end{aligned}
$$

or equivalently

$$
\begin{aligned}
& \xi=\xi^{\prime} \cos \alpha+\zeta^{\prime} \sin \alpha \\
& \zeta=-\xi^{\prime} \sin \alpha+\zeta^{\prime} \cos \alpha
\end{aligned}
$$

Pluging Eq. (B.1) into Eq. (B.4), we have

$$
\xi^{\prime}=\xi \cos \alpha+H_{\text {box }} \sin \alpha .
$$

The tip radius of the dendrite is $\rho$ and the dendrite in coordinate system $\left(\xi^{\prime}, \eta^{\prime}, \zeta^{\prime}\right)$ is an elliptic paraboloid opening downward along the $\zeta^{\prime}$-axis and it is given by

$$
\zeta^{\prime}=-\left(\frac{\xi^{2}+\eta^{\prime 2}}{2 \rho}\right) .
$$

Combining Eqs (B.1), (B.7) and (B.9) yields

$$
-H_{\mathrm{box}}=-\xi^{\prime} \sin \alpha-\left(\frac{\xi^{\prime 2}+\eta^{\prime 2}}{2 \rho}\right) \cos \alpha .
$$

We rewrite Eq. (B.10) as

$$
\left(\xi^{\prime}+\rho \tan \alpha\right)^{2}+\eta^{\prime 2}=W^{2}
$$

where

$$
W=\sqrt{(\rho \tan \alpha)^{2}+\frac{2 \rho H_{\mathrm{box}}}{\cos \alpha}} .
$$

Using the fact that $\eta=\eta^{\prime}$ and Eq. (B.8), Eq. (B.11) is rewritten as

$$
\left(\xi \cos \alpha+H_{\mathrm{box}} \sin \alpha+\rho \tan \alpha\right)^{2}+\eta^{2}=W^{2}
$$

We can show the intersected region is an ellipse given by the following equation:

$$
\left(\frac{\bar{\xi}}{a}\right)^{2}+\left(\frac{\eta}{b}\right)^{2}=1
$$

where

$$
\begin{aligned}
\bar{\xi} & =\xi+H_{\text {box }} \tan \alpha+\rho \frac{\tan \alpha}{\cos \alpha} \\
a & =\frac{W}{\cos \alpha} \\
b & =W
\end{aligned}
$$

Finally, we find:

$$
\mathcal{A}_{s}=\pi a b=\pi \frac{\left[(\rho \tan \alpha)^{2}+\frac{2 \rho H_{\mathrm{box}}}{\cos \alpha}\right]}{\cos \alpha}
$$




\section{References}

[1] A. Karma, W.-J. Rappel, Quantitative phase-field modeling of dendritic growth in two and three dimensions, Physical Review E 57 (4) (1998) 4323. doi:10.1103/PhysRevE.57.4323.

[2] W. Boettinger, J. Warren, C. Beckermann, A. Karma, Phase-field simulation of solidification, Ann. Rev. Mater. Res. 32 (1) (2002) 163-194. doi:10.1146/annurev.matsci.32.101901.155803.

[3] A. Karma, D. Tourret, Atomistic to continuum modeling of solidification microstructures, Current Opinion in Solid State and Materials Science 20 (1) (2016) 25-36. doi:10.1016/j.cossms.2015.09.001.

[4] C. Wang, C. Beckermann, A multiphase solute diffusion model for dendritic alloy solidification, Metall. Trans. A 24A (1993) 2787-2802. doi:10.1007/BF02659502.

[5] C. Wang, C. Beckermann, Prediction of columnar to equiaxed transition during diffusion-controlled denditric alloy solidification, Metall. Mater. Trans. A 25 (1994) 1081-1093. doi:10.1007/BF02652282.

[6] M. Martorano, C. Beckermann, C. Gandin, A solutal interaction mechanism for the columnar-toequiaxed transition in alloy solidification, Metall. Mater. Trans. A 34 (2003) 1657-1674. doi:10.1007/s11661-003-0311-X.

[7] C. Gandin, M. Rappaz, A coupled finite elementcellular automaton model for the prediction of dendritic grain structures in solidification processes, Acta Metall. Mater. 42 (7) (1994) 2233-2246. doi:10.1016/0956-7151(94)90302-6.

[8] M. Vandyoussefi, A. Greer, Application of cellular automaton-finite element model to the grain refinement of directionally solidified Al4.15 wt\% Mg alloys, Acta Mater. 50 (2002) 1693-1705. doi:10.1016/S1359-6454(02)00015-0.

[9] P.-A. Geslin, C.-H. Chen, A. Molavi Tabrizi, A. Karma, Dendritic Needle Network Modeling of the Columnar-to-Equiaxed Transition. Part I: Two Dimensional Formulation and Comparison with Theory, Acta Materialia.

[10] D. Tourret, A. Karma, Multiscale dendritic needle network model of alloy solidification, Acta Materialia 61 (17) (2013) 6474-6491. doi:10.1016/j.actamat.2013.07.026.
[11] D. Tourret, A. J. Clarke, S. D. Imhoff, P. J. Gibbs, J. W. Gibbs, A. Karma, Three-dimensional multiscale modeling of dendritic spacing selection during Al-Si directional solidification, JOM 67 (8) (2015) 1776-1785. doi:10.1007/s11837-015-1444-2.

[12] D. Tourret, A. Karma, A. J. Clarke, P. J. Gibbs, S. D. Imhoff, Three-dimensional dendritic needle network model with application to Al-Cu directional solidification experiments, in: IOP Conference Series: Materials Science and Engineering, Vol. 84, 2015, p. 012082. doi:10.1088/1757-899X/84/1/012082.

[13] D. Tourret, A. Karma, Three-dimensional dendritic needle network model for alloy solidification, Acta Materialia 120 (2016) 240-254. doi:10.1016/j.actamat.2016.08.041.

[14] G. Zimmermann, L. Sturz, B. Billia, N. MangelinckNoel, H. N. Thi, C.-A. Gandin, D. J. Browne, W. U. Mirihanage, Investigation of columnar-to-equiaxed transition in solidification processing of AlSi alloys in microgravity-the CETSOL project, in: Journal of Physics: Conference Series, Vol. 327, 2011, p. 012003. doi:10.1088/1742-6596/327/1/012003.

[15] G. Zimmermann, L. Sturz, B. Billia, N. MangelinckNoel, D. R. Liu, H. Nguyen Thi, N. Bergeon, C.-A. Gandin, D. J. Browne, C. Beckermann, et al., Columnar-to-equiaxed transition in solidification processing of AlSi7 alloys in microgravity the CETSOL project, in: Materials Science Forum, Vol. 790, Trans Tech Publ, 2014, pp. 12-21. doi:10.4028/www.scientific.net/MSF.790-791.12.

[16] G. Zimmermann, L. Sturz, H. Nguyen-Thi, N. Mangelinck-Noël, Y. Li, C.-A. Gandin, R. Fleurisson, G. Guillemot, S. Mcfadden, R. P. Mooney, et al., Columnar and Equiaxed Solidification of Al-7 wt.\% Si Alloys in Reduced Gravity in the Framework of the CETSOL Project, JOM 69 (8) (2017) 1269-1279. doi:10.1007/s11837-017-23974.

[17] D. R. Liu, N. Mangelinck-Noël, C.-A. Gandin, G. Zimmermann, L. Sturz, H. N. Thi, B. Billia, Structures in directionally solidified Al-7wt.\% $\mathrm{Si}$ alloys: Benchmark experiments under microgravity, Acta Materialia 64 (2014) 253-265. doi:10.1016/j.actamat.2013.10.038.

[18] W. Kurz, D. Fisher, Fundamentals of Solidification, (3rd Edition) Trans Tech Publications, Switzerland, 1992. 
[19] A. Karma, Phase-field formulation for quantitative modeling of alloy solidification, Physical Review Letters 87 (11) (2001) 115701. doi:10.1103/PhysRevLett.87.115701.

[20] W. Kurz, B. Giovanola, R. Trivedi, Theory of microstructural development during rapid solidification, Acta Metallurgica 34 (5) (1986) 823-830. doi:https://doi.org/10.1016/0001-6160(86)90056-8.

[21] J. Dantzig, M. Rappaz, Solidification, Engineering Sciences, EPFL press, 2009.

URL http://www. solidification.org/

[22] A. Bogno, H. Nguyen-Thi, G. Reinhart, B. Billia, J. Baruchel, Growth and interaction of dendritic equiaxed grains: In situ characterization by synchrotron X-ray radiography, Acta Materialia 61 (4) (2013) 1303-1315. doi:10.1016/j.actamat.2012.11.008.

[23] J. Langer, Chance and Matter: Lectures on the Theory of Pattern Formation, Les Houches, Session XLVI, edited by J. Souletie, J. Vannimenus, and R. Stora (North-Holland, Amsterdam, 1987) 629-711.

[24] J. Langer, Dendrites, viscous fingers, and the theory of pattern formation, in: Dynamics and Patterns in Complex Fluids, Springer, 1990, pp. 190-193.

[25] A. Barbieri, J. Langer, Predictions of dendritic growth rates in the linearized solvability theory, Physical Review A 39 (10) (1989) 5314. doi:10.1103/PhysRevA.39.5314.

[26] J. R. Morris, Complete mapping of the anisotropic free energy of the crystal-melt interface in al, Physical Review B 66 (14) (2002) 144104. doi:10.1103/PhysRevB.66.144104.

[27] M. Plapp, A. Karma, Multiscale random-walk algorithm for simulating interfacial pattern formation, Physical Review Letters 84 (8) (2000) 1740. doi:10.1103/PhysRevLett.84.1740.

[28] G. Ivantsov, Temperature field around a spherical, cylindrical, and needle-shaped crystal, growing in a pre-cooled melt, Dokl. Akad. Nauk. SSSR 58 (1947) 567-569.

[29] C.-A. Gandin, From constrained to unconstrained growth during directional solidification, Acta Materialia 48 (10) (2000) 2483-2501. doi:10.1016/S1359-6454(00)00070-7.
[30] D. Tourret, M. Francois, A. Clarke, Multiscale dendritic needle network model of alloy solidification with fluid flow, Computational Materials Science 162 (2019) 206-227. doi:10.1016/j.commatsci.2019.02.031. 